\title{
EXISTENCE AND UNIQUENESS OF MINIMAL GRAPHS IN HYPERBOLIC SPACE*
}

\author{
RICARDO SA EARP ${ }^{\dagger}$ AND ERIC TOUBIANA ${ }^{1}$
}

\begin{abstract}
In this paper we prove general existence and uniqueness theorems for minimal vertical graphs in hyperbolic space.
\end{abstract}

Introduction. In this paper we shall prove general existence and uniqueness results for vertical minimal graphs in the hyperbolic space $\mathbf{H}^{3}=\left\{\left(x_{1}, x_{2}, x_{3}\right) \in\right.$ $\left.\mathbf{R}^{3}, x_{3}>0\right\}$ over bounded and unbounded domains in $\partial_{\infty} \mathbf{H}^{3}$ (see Eq. 1-1). We solve the Dirichlet problem for the minimal equation (Problem $(P)$ in $\S 1$ ) in two variables $x_{1}, x_{2}$ over bounded $C^{0}$ convex domains, given arbitrary non negative continuous boundary value data (see Theorem 2.3). If the boundary data and the domain are smooth enough an analogous result also holds in every dimension (See Theorem 2.2).

The main result of this paper is the derivation of the Perron process for the minimal vertical equation in the upper halfspace model of hyperbolic three space (see Problem $(P)$ in $\S 1)$. More precisely, we shall infer that existence of a supersolution yields a solution for the Dirichlet Problem $(P)$. When the boundary $\partial \Omega$ is $C^{0}$ convex at $p$, or the boundary data $f$ vanish at $p$, and $p$ has a barrier (see Definition 1.6) then the solution given by Perron process is continuous up to $p$ and takes the given boundary data at $p$. In particular, in any convex $\operatorname{arc} \Gamma \subset \partial \Omega$ such a solution is continuous up to $\Gamma$. Moreover, if $p$ has a barrier and $f \geq \alpha>0$ then the solution given by Perron process is continuous up to the boundary and takes the prescribed boundary value $f$ at $p$ (see Theorem 3.4). We shall infer by Perron process, several existence and uniqueness results for minimal complete and non complete graphs over $C^{0}$ unbounded convex and non convex domains, namely complete minimal graphs, invariant by a given 1-parameter group of hyperbolic or parabolic isometries of $\mathbf{H}^{3}$ (see Propositions 3.9 and 3.12). We shall also infer from Perron process the existence of the Dirichlet problem for the minimal vertical equation over a band, given arbitrary non negative continuous boundary data (see Theorem 4.1). Theoretically, this result can be viewed as an example of application of Perron process. For a convex unbounded domain in a band $\Omega$, we shall prove the following uniqueness result: if the prescribed boundary data $f$ is bounded, non negative and uniformly continuous then the Dirichlet problem for the minimal vertical equation admits at most one solution. Moreover, any such a solution is bounded and uniformly continuous. In fact this result holds in higher dimensions (see Corollary 1.11 and Remark 1.9). From Perron process we shall deduce a maximum principle for domains in a proper sector (see Theorem 4.3) that gives rise to an existence result for the Dirichlet problem $(P)$ over convex domains in a proper sector (see Theorem 4.5 and Remark 4.7). Next, we explain the main tools.

Throughout this paper, we use L. Simon interior estimates for mean curvature type equations in two variables as a major tool, see [Si], together with the control at

* Received November 11, 1998; accepted for publication November 30, 1999.

† Pontifícia Universidade Católica, Rio De Janeiro. Departamento de Matemática, Rua Marquês De São Vicente 225, 22 453-900 Rio De Janeiro, Brazil (earp@mat.puc-rio.br). The author was partially suported by CNPq and PRONEX of Brazil and the second author was supported by FAPERJ of Brazil.

${ }^{1}$ Université Paris VII, Département de Mathématiques. 2, Place Jussieu, 75251 Paris Cedex 05, France (toubiana@math.jussieu.fr). The author is grateful to the Mathematics Department of PUC-Rio, Brazil, for their hospitality during the elaboration of this paper. 
the boundary that we have succeeded (see Theorem 1.7) to establish the mentioned Perron process, see $\S 3$, for the minimal vertical equation, Eq. 1-1. Of course, we also make use of the geometry of hyperbolic space to get some useful boundary and interior a priori estimates.

There are earlier results related to this article that we now mention. Firstly, we must note the pioneer work of Anderson about existence results for complete minimal varieties in hyperbolic space with preassigned asymptotic boundary, see for instance [An, 1] and [An, 2]. In [An, 1], Anderson solved for a $C^{2}$ bounded mean convex domain $\Omega$ in $\mathbf{R}^{n}, n \geq 2$, the Dirichlet problem for the minimal vertical equation (Problem $(P))$ with zero boundary data. Secondly, it is worth mentioning that $\mathrm{Lin}$ in $[\mathrm{Li}, 1]$ gave a short proof of Anderson's existence result cited above using PDE methods. He also proved in the same paper uniqueness, and he achieved his main result about regularity of the solution up to the boundary; that is, if the boundary $\partial \Omega$ is smooth then the graph is smooth as $\partial \Omega$ up to the boundary. In [Li, 2] Lin studied the asymptotic behavior of area-minimizing locally rectifiable currents in hyperbolic space. Recently, the authors have carried out an existence result about minimal vertical graphs over an annular domain, see [SE-To, 1].

Now, by way of conclusion, we are going to state two open questions arising from this work. An interesting question is related to the maximum principle at infinity for minimal surfaces in Euclidean space inferred by Langevin and Rosenberg in [La-Ro], Meeks and Rosenberg in [Me-Ro] and Soret in [So]. Is it true that if $M_{1}$ and $M_{2}$ are two minimal vertical graphs over an unbounded domain $\Omega$ such that $\operatorname{dist}\left(\partial M_{1}, M_{2}\right)>0$ and $\operatorname{dist}\left(\partial M_{2}, M_{1}\right)>0$ then $\operatorname{dist}\left(M_{2}, M_{1}\right)>0$ ?

Bers showed that the classical minimal surface equation in Euclidean space cannot have an isolated singularity (see [Be]). It is not difficult to show that if $u$ is a solution of the minimal vertical equation (Eq. 1-1) over a puncture disk then $u$ is bounded from above (on a possible smaller puncture disk) by a positive constant $b$ and bounded from bellow by a positive constant $a$. The proof of this assertion uses minimal hyperbolic catenoids as suitable barriers. Thus one can naturally ask if $u$ extends smoothly to the puncture?

1. Basic results. In this section we give some technical tools and we prove some basic results that we shall need throughout this paper. We choose the half-space model of hyperbolic space. Namely

$$
\mathbf{H}^{\mathbf{n}+1}=\left\{\left(x_{1}, \ldots, x_{n+1}\right) \in \mathbf{R}^{n+1}, x_{n+1}>0\right\}
$$

equipped with the hyperbolic metric

$$
d s^{2}=\frac{d x_{1}^{2}+\ldots d x_{n+1}^{2}}{x_{n+1}^{2}} .
$$

We are concerned with positive $C^{2}$ functions $u$ defined on domains $\Omega$ of $\mathbf{R}^{n}=\left\{x_{n+1}=0\right\}$ (considered as asymptotic boundary of $\mathbf{H}^{\mathbf{n}+\mathbf{1}}$ ) whose graph $S=\left\{\left(x_{1}, \ldots, x_{n}, u\left(x_{1}, \ldots, x_{n}\right)\right),\left(x_{1}, \ldots, x_{n}\right) \in \Omega\right\}$ is a minimal hypersurface of $\mathbf{H}^{\mathbf{n}+\mathbf{1}}$. We say that $S$ is a vertical graph. That is, we consider $C^{2}$ functions $u$ on $\Omega$ satisfying the following strictly elliptic quasilinear PDE:

$$
\mathcal{D}(u)=\sum_{i, j=1}^{n}\left(\delta_{i j}-\frac{u_{i} u_{j}}{\left(1+|D u|^{2}\right)}\right) u_{i j}+\frac{n}{u}=0
$$

in $\Omega$. We will allow that $u$ takes zero value boundary data on a part of $\partial \Omega$. Notice 
that $\mathcal{D}(u)-\frac{n}{u}=0$ is the minimal hypersurface equation in euclidean space $\mathbf{R}^{n+1}$.

TheOREM 1.1. Let $\Omega \subset \mathbf{R}^{n}$ be a bounded domain such that $\partial \Omega \in C^{2, \alpha}$. Let $\phi \in C^{2, \alpha}(\bar{\Omega})$ and let $\mathcal{Q}$ be a quasilinear strictly elliptic operator in $\bar{\Omega}$. Consider the Dirichlet problem

$$
\mathcal{Q} u=0 \text { in } \Omega \text { and } u=\phi \text { on } \partial \Omega \text {. }
$$

For $t \in[0,1]$ consider the family of Dirichlet problems:

$$
\begin{aligned}
\mathcal{Q}_{t} u & =a_{i j}(x, u, D u) D_{i j} u+b(x, u, D u, t)=0, \text { in } \Omega \\
u & =t \phi \text { on } \partial \Omega
\end{aligned}
$$

satisfying:

(i) $\mathcal{Q}_{1}=\mathcal{Q}$ and $b(x, u, D u, 0)=0$;

(ii) The operators $\mathcal{Q}_{t}$ are strictly elliptic on $\bar{\Omega}$ for all $t \in[0,1]$;

(iii) The coefficients $a_{i j} \in C^{1}\left(\bar{\Omega} \times \mathbf{R} \times \mathbf{R}^{n}\right), b \in C^{\alpha}\left(\bar{\Omega} \times \mathbf{R} \times \mathbf{R}^{n}\right)$ for each $t$ and considering as mapping from $[0,1]$ into $C^{\alpha}\left(\bar{\Omega} \times \mathbf{R} \times \mathbf{R}^{n}\right)$, the function $b$ is continuous. Suppose there exists a constant $A$ such that for each $t$ and for each $C^{2, \alpha}(\bar{\Omega})$ solution $u$ of the Dirichlet problem $\mathcal{Q}_{t} u=0$ in $\Omega$ and $u=t \phi$ on $\partial \Omega, 0 \leq t \leq 1$, we have :

$$
|u|_{C^{1}(\bar{\Omega})}=\sup _{\Omega}|u|+\sup _{\Omega}|D u|<A
$$

( $A$ independent of $u$ and $t$ ). Then the Dirichlet problem $(*)$ is solvable in $C^{2, \alpha}(\bar{\Omega})$.

The above existence theorem is a consequence of Schauder theory and the global Hölder estimate of Ladyzhensaya and Ural'tseva, see [Gi-Tr, Theorem 13.7], and [GiTr, Theorem 11.8].

The next Lemma is an adaptation of a result of $\mathrm{L}$. Simon, see [Si, Theorem 2"].

LEMMA 1.2. Let $\Omega \subset \mathbf{R}^{2}$ be a domain and let $\left.u: \Omega \rightarrow\right] 0,+\infty\left[\right.$ be a $C^{2}$ solution of (Eq. 1-1) (that is the graph of $u$ is a minimal surface in $\mathbf{H}^{3}$ ). Let $p \in \Omega$. Then there exists $\rho>0$ such that $\overline{D_{\rho}(p)} \subset \Omega$ (where $D_{\rho}(p)$ is the euclidean open disk of radius $\rho$ centered at $p$ ), and there exist two real constants $c_{1}, c_{2}$ depending only of $\rho$ and $\inf _{D_{\rho}(p)} u(x)$ such that:

$$
|D u(p)| \leq c_{1} \exp \left(c_{2} \frac{u(p)}{\rho}\right) .
$$

Consequently for each compact part $K \subset \Omega$ there exists a real number $M>0$ depending only of $\inf _{K}(u)$ and $\sup _{K}(u)$ such that:

$$
|D u(p)|<M
$$

for each $p \in K$.

Proof. We are going to use results and notations of L. Simon [Si]. The function $u$ satisfies (Eq.1-1) on $\Omega$. For any point $(x, z, p) \in \Omega \times \mathbf{R} \times \mathbf{R}^{2}$, we define $a_{i j}(x, z, p)=$ $\delta_{i j}-p_{i} p_{j} /\left(1+p_{1}^{2}+p_{2}^{2}\right), i, j=1,2$. Let $\delta>0$ be such as the conditions (3.2) of [Si] are satisfied where the functions $a_{i j}^{*}$ are defined by $(2.1)$ of [Si]. Note that they are the same functions which appear in the equation of minimal surface of $\mathbf{R}^{3}$. Let $p \in \Omega$ and let $r>0$ such that $\overline{D_{r}(p)} \subset \Omega$. Let $\alpha=\inf _{D_{r}(p)} u(x)$, observe that $\alpha>0$. Let $h: \mathbf{R} \rightarrow \mathbf{R}^{+}$be any $C^{1}$ positive function such that:

(1) $h(z)=\frac{1}{z}$ for any $z \geq \alpha$, 
(2) $h^{\prime}<0$,

(3) $h<\frac{2}{\alpha}$.

Then $u$ satisfies on $D_{r}(p)$ the PDE

(**) $\frac{1+u_{2}^{2}}{1+u_{1}^{2}+u_{2}^{2}} u_{11}-2 \frac{u_{1} u_{2}}{1+u_{1}^{2}+u_{2}^{2}} u_{12}+\frac{1+u_{1}^{2}}{1+u_{1}^{2}+u_{2}^{2}} u_{22}=-2 h(u)=b(x, u, D u)$.

Now $b(x, z, p)$ is a $C^{1}$ function on $D_{r}(p) \times \mathbf{R} \times \mathbf{R}^{2}$ and we can apply Theorem $2^{\prime \prime}$ of [Si]. For this observe that setting $b^{*}=\left(1+|p|^{2}\right)^{-1 / 2} b$, the condition (3.10) of [Si] is satisfied provide that $2 h(u)<\frac{\delta}{r}$ (since $h^{\prime}<0$ and $\frac{\partial b^{*}}{\partial x_{j}}=0, j=1,2$ ). Then for any positive $\rho \leq r$ such that $\frac{4}{\alpha}<\frac{\delta}{\rho}$, Theorem $2^{\prime \prime}$ of [Si] shows the existence of constants $c_{1}$ and $c_{2}$ such that $(*)$ holds. Note that the condition (1.3) of [Si] holds on $D_{\rho}(p)$ with $\mu=\frac{4}{\alpha}$. The other assertion follows immediately.

THEOREM 1.3. (Compactness theorem) Let $\Omega \subset \mathbf{R}^{2}$ be a domain and let $\left(u_{n}\right)_{n \in \mathrm{N}}$ be a sequence of $C^{2}$ functions on $\Omega$ satisfying (Eq. 1-1). Suppose that for each compact part $K \subset \Omega$, there exist two positive real numbers $a, b$ (depending only of $K$ ) such that $0<a<u_{n}(x)<b$ for every $x \in K$ and every $n$.

Then there exists a subsequence of $\left(u_{n}\right)$ converging on $K$, in the $C^{2}$ topology, to a $C^{2}$ function $u$ satisfying (Eq.1-1) on $K$. Consequently there exists a subsequence of $\left(u_{n}\right)$ converging on $\Omega$, in the $C^{2}$ topology, to a $C^{2}$ function $u$ satisfying (Eq.1-1) on $\Omega$.

Proof. Let $K \subset \Omega$ be a compact part. We infer from Lemma 1.2 the existence of a real number $M>0$ such that

$$
\left|D u_{n}(x)\right|<M
$$

for every $x \in K$ and every $n$. Then using interior Hölder estimate of Ladyzhenskaya and Ural'tseva, see Theorem 13.1 of [Gi-Tr], we get uniform $C^{1, \alpha}$ estimate for the sequence $u_{n}$ on $K$. At last, using Schauder theory ([Gi-Tr] Corollary 6.3) we have uniform $C^{2, \alpha}$ estimate on $K$. Therefore the sequence $u_{n}$ with their first and second derivatives on $K$ give rise to a normal family. Then there exists a subsequence converging on $K$ to a $C^{2}$ function in the $C^{2}$ topology. By a continuity argument, this function satisfies (Eq.1-1). Considering a compact exhaustion of $\Omega$ and using the standard diagonal process, we infer the existence of a subsequence converging on $\Omega$ in the $C^{2}$ topology to a solution of (Eq.1-1).

Now we write down the well-known Maximum Principle.

Proposition 1.4. (Maximum Principle) Let $\Omega \subset \mathbf{R}^{n}$ be a bounded domain. Let $u, v \in C^{2}(\Omega) \cap C^{0}(\bar{\Omega})$ be two positive functions. Assume that $u_{\mid \partial \Omega} \leq v_{\mid \partial \Omega}$ and that $\mathcal{D}(u) \geq \mathcal{D}(v)$ on $\Omega$.

(i) Interior Maximum Principle: Then we have $u \leq v$ on $\Omega$ and equality occurs at an interior point if and only if $u=v$.

(ii) Boundary Maximum Principle: Let $p \in \partial \Omega$ be such that $u(p)=v(p)$. Suppose that $p$ lies on the boundary of a round ball contained in $\Omega$. Assume that the outward derivative $\frac{\partial(u-v)}{\partial \nu}$ exists at $p$. Then we have

$$
\frac{\partial(u-v)}{\partial \nu}(p)>0
$$


unless $u=v$ on $\Omega$.

Proof. We will proceed the proof for the sake of completeness. A straightforward computation shows that:

$$
\begin{aligned}
& \left(1+|D u|^{2}\right) \mathcal{D}(u)-\left(1+|D v|^{2}\right) \mathcal{D}(v)=\sum_{i, j=1}^{n}\left[\delta_{i j}\left(1+|D u|^{2}\right)-u_{i} u_{j}\right]\left(u_{i j}-v_{i j}\right) \\
& +\sum_{k=1}^{n}\left[\left(u_{k}+v_{k}\right)\left(\frac{n}{v}+\sum_{i, j=1}^{n} \delta_{i j} v_{i j}\right)-\sum_{i=1}^{n}\left(u_{i}+v_{i}\right) v_{i k}\right]\left(u_{k}-v_{k}\right)-\frac{n\left(1+|D u|^{2}\right)}{u v}(u-v) .
\end{aligned}
$$

We define a linear operator $\mathcal{L}$ setting:

$$
\begin{aligned}
\mathcal{L}(f) & =\sum_{i, j=1}^{n}\left[\delta_{i j}\left(1+|D u|^{2}\right)-u_{i} u_{j}\right] f_{i j} \\
& +\sum_{k=1}^{n}\left[\left(u_{k}+v_{k}\right)\left(\frac{n}{v}+\sum_{i, j=1}^{n} \delta_{i j} v_{i j}\right)-\sum_{i=1}^{n}\left(u_{i}+v_{i}\right) v_{i k}\right] f_{k}-\frac{n\left(1+|D u|^{2}\right)}{u v} f .
\end{aligned}
$$

for every $C^{2}$ positive function $f$ on $\Omega$. Let us observe that $\mathcal{L}$ is an uniformly elliptic linear operator of the following form: $\sum_{i, j} a_{i j}(x) D_{i j} f+\sum_{k} b_{k}(x) D_{k} f+h(x) f$ where $h(x) \leq 0$ on $\Omega$. It follows that $\mathcal{L}$ satisfies the Maximum Principle as stated in [Pr-We, Theorem 6 p.64]. As $\mathcal{L}(u-v) \geq 0$ on $\Omega$ and $u_{\mid \partial \Omega}-v_{\mid \partial \Omega} \leq 0$ we conclude that $u-v \leq 0$ on $\Omega$ with equality at an interior point if and only if $u=v$. This shows the Interior Maximum Principle.

Furthermore observe that in the conditions of (ii) we can apply Theorem 8, p. 67 in $[\mathrm{Pr}-\mathrm{We}]$ to infer the Boundary Maximum Principle.

REMARK 1.5. 1) In the same way, differentiating (Eq.1-1) with respect to $x_{q}$, we find that $u_{q}$ satisfies an uniformly elliptic linear operator of the following form: $\sum_{i, j} a_{i j}(x) D_{i j} u_{q}+\sum_{k} b_{k}(x) D_{k} u_{q}+h(x) u_{q}$ where $h(x) \leq 0$ on $\Omega$. We conclude that $\left|u_{q}\right|$ and then $|D u|$ has not an interior maximum. In particular if a solution $u$ of (Eq $1-1)$ is $C^{1}$ up to the boundary, the maximum of $|D u|$, if any, is assumed on $\partial \Omega$.

2) As a direct consequence we get that, in the same conditions of Proposition 1.4, if $u=v$ on $\partial \Omega$ and $\mathcal{D}(u)=\mathcal{D}(v)=0$ on $\Omega$ then $u=v$ on $\bar{\Omega}$. That is the Dirichlet problem for vertical minimal graph in $\mathbf{H}^{n+1}$ admits at most one solution on bounded domains.

3) Let $\Omega \subset \mathbf{R}^{n}$ be a compact domain. Consider $M_{1}, M_{2} \subset \mathbf{H}^{n+1}$ two hypersurfaces which are graph over $\bar{\Omega}$. Suppose that $M_{1}$ is an euclidean minimal hypersurface, $M_{2}$ is a hyperbolic minimal hypersurface and $\partial M_{1}$ stays below $\partial M_{2}$. Then it immediately follows from the Maximum Principle that $M_{1}$ stays below $M_{2}$ without any interior contact point. Note that $\partial M_{1}$ and $\partial M_{2}$ can have contact.

4) Let $\Omega \subset \mathbf{R}^{n}$ be a $C^{2}$ domain and consider two positive $C^{2}$ functions $u$ and $v$ on $\Omega$ satisfying (Eq.1-1) and such that $u \leq v$. Let $p \in \partial \Omega$ be a boundary point such that $u(p)=v(p), u$ and $v$ are $C^{1}$-continuous up to $p$ and such that $u_{i}(p)=v_{i}(p)$ for $i=1, \ldots, n$. Then the Boundary Maximum Principle implies that $u=v$ on $\Omega$. Geometrically this means the following. Let $M_{1}, M_{2} \subset \mathbf{H}^{n+1}$ be two minimal hypersurfaces with $C^{2}$ boundaries. Assume that $M_{1}$ and $M_{2}$ are $C^{1}$ up to a common 
boundary point $p$. Suppose that $M_{1}$ and $M_{2}$ are tangent at $p, M_{2}$ stays above $M_{1}$ in a neighborhood $V \subset \mathbf{H}^{n+1}$ of $p$ and the tangent spaces of the two boundaries $\partial M_{1}$ and $\partial M_{2}$ agree at $p$. Then the two hypersurfaces are equal in a neighborhood $V$ of $p$.

Definition 1.6. Let $\Omega \subset \mathbf{R}^{n}$ be a domain. Let $f: \partial \Omega \rightarrow[0,+\infty[$ be a continuous function. Consider the following Dirichlet problem $(P)$ :

$$
\left\{\begin{aligned}
\sum_{i, j=1}^{n}\left(\delta_{i j}-\frac{u_{i} u_{j}}{1+|D u|^{2}}\right) u_{i j}+\frac{n}{u} & =0 \text { on } \Omega \\
u_{\mid \partial \Omega} & =f \\
u & \in C^{2}(\Omega) \cap C^{0}(\bar{\Omega})
\end{aligned}\right.
$$

1) Let $p \in \partial \Omega$ be a boundary point. Assume first that $f(p)>0$. Suppose that there is an open neighborhood $\mathcal{N}$ of $p$ in $\mathbf{R}^{n}$ such that for any $M>0$ (resp. $n \in \mathbf{N}^{*}$ ) there exist a sequence of functions $\omega_{k}^{+}$(resp. $\left.\omega_{k}^{-}\right)$in $C^{2}(\mathcal{N} \cap \Omega) \cap C^{0}(\overline{\mathcal{N} \cap \Omega)}$ such that

(i) $\omega_{k}^{+}(x)_{\mid \partial \Omega \cap \mathcal{N}} \geq f(x)$ and $\omega_{k}^{+}(x)_{\mid \partial \mathcal{N} \cap \Omega} \geq M$ (resp. $\omega_{k}^{-}(x)_{\mid \partial \Omega \cap \mathcal{N}} \leq f(x)$ and $\omega_{k}^{-}(x)_{\mid \partial \mathcal{N} \cap \Omega} \leq \frac{1}{n}$ )

(ii) $\mathcal{D}\left(\omega_{k}^{+}\right) \leq 0$ (resp. $\mathcal{D}\left(\omega_{k}^{-}\right) \geq 0$ in the part where $\omega_{k}^{-}>0$ ) in $\mathcal{N} \cap \Omega$,

(iii) $\lim _{k \rightarrow+\infty} \omega_{k}^{+}(p)=f(p)$ (resp. $\left.\lim _{k \rightarrow+\infty} \omega_{k}^{-}(p)=f(p)\right)$. If $f(p)=0$ we substitute $\omega_{k}^{-}(x)$ for the vanishing function on $\mathcal{N}$.

Then we say that $p$ admits a superior (resp. inferior) barrier for the problem $(P)$. If $p$ admits a superior and an inferior barrier we say more shortly that $p$ admits a barrier.

2) Let $u: \bar{\Omega} \rightarrow \mathbf{R}^{+}$be a solution of the problem $(P), u \in C^{2}(\Omega) \cap C^{0}(\bar{\Omega})$. We say that $u$ has a modulus of continuity along $\partial \Omega$ if for every $\varepsilon>0$, there is $\delta>0$ such that if $|p-x|<\delta, p \in \partial \Omega, x \in \Omega$, then $|u(p)-u(x)|<\varepsilon$.

THEOREM 1.7. Let $\Omega \subset \mathbf{R}^{2}$ be a $C^{0}$ convex domain in a band (a band is a domain bounded by two parallel straight lines of $\mathbf{R}^{2}$ ). Let $f$ be a non negative and bounded function on $\partial \Omega$. Then there exist a real number $B>0$ such that $0<u \leq B$ on $\Omega$ for any solution of the problem $(P)$. Furthermore, if $f$ is also uniformly continuous, then any solution of $(P)$ has a modulus of continuity along $\partial \Omega$.

Proof. Let $A \geq 0$ be an upper bound for $f$. Consider a hyperbolic catenoid $C$ in $\mathbf{H}^{3}$ such that $\partial_{\infty} C$ (which is composed of two circles) has a component on each side of the band in $\mathbf{R}^{2}$. Up to a homothety with respect to a point of $\partial_{\infty} \mathbf{H}^{3}=\mathbf{R}^{2}$, which is a hyperbolic isometry, we can assume that the height of $C$ above the boundary of the band is greatest than $A$. Therefore $C$ is above the graph of $f$ along $\partial \Omega$. Observe that if $v$ is a $C^{2}$ function satisfying $\mathcal{D}(v)=0$, then $\mathcal{D}(v+a)<0$ for any $a>0$. By the Maximum Principle (Proposition 1.4), using euclidean vertical translations on $C$, we deduce that $C$ is above the graph of any solution of $(P)$. Using the horizontal translations parallel to the band (they are hyperbolic isometries), we conclude that each translated of $C$ is above the graph of any solution of $(P)$. Therefore there exists a real number $B>0$ such that $u<B$ for any solution of $(P)$. Next we will construct a barrier at any point of $\partial \Omega$ using a construction done in [Ro-SE].

Let $p \in \partial \Omega$ and let $L$ be any straight line through $p$ keeping $\Omega$ on one side of $L$. Let $\varepsilon>0$ and let $\delta_{0}>0$ be such that $|f(p)-f(q)|<\varepsilon / 3$ if $|p-q|<\delta_{0}, p, q \in \partial \Omega$. 
Define

$$
\varphi(x)=f(p)+\frac{\varepsilon}{3}+2 B \frac{\log \left(1+|p-x|^{2}\right)}{\log \left(1+\delta_{0}\right)}
$$

We have $f \leq \varphi$ on $\partial \Omega$ and $|D \varphi|,\left|D^{2} \varphi\right|$ are bounded where $\left|D^{2} \varphi\right|=\sum_{i j} \sup _{x}\left|D_{i j} \varphi(x)\right|$. Define $d(x)=d(x, L)$ and $v(x)=\psi(d(x))=\frac{1}{b} \log \left(1+\frac{e^{2 B b}-1}{\delta_{1}} d(x)\right)$ where $x \in \Omega$ and $b$ and $\delta_{1}$ are two positive real numbers to be defined later. In order to evaluate $\mathcal{D}(\varphi+v)$ we set $\Lambda=\Lambda(x)=1+|D \varphi+D v|^{2}$ and $a_{i j}(x)=\delta_{i j} \Lambda-D_{i}(\varphi+v) D_{j}(\varphi+v)$. Note that for any vector $\xi=\left(\xi_{1}, \xi_{2}\right)$ and for any $C^{2}$ function $h$ we have:

$$
|\xi|^{2} \leq \sum_{i, j} a_{i j} \xi_{i} \xi_{j} \leq \Lambda|\xi|^{2} \text {, and } \sum_{i, j} a_{i j} D_{i j} h \leq \Lambda\left|D^{2} h\right|
$$

Define $\mathcal{F}=\sum_{i j} a_{i j} D_{i} v D_{j} v$. As $\varphi$ is $C^{1}$ bounded and $C^{2}$ bounded there is $\alpha, \beta>1$ such that $\Lambda<\alpha \mathcal{F}$ and $\Lambda\left|D^{2} \varphi\right|<\beta \mathcal{F}$ whenever $|D v| \geq 1$. Observe that $\frac{\psi^{\prime \prime}}{\left(\psi^{\prime}\right)^{2}}=-b$, $D_{i} v=\psi^{\prime}(d) D_{i} d$ and $D_{i j} v=\psi^{\prime \prime}(d) D_{i} d D_{j} d$ since $D_{i j} d=0$. From this we infer that

$$
\begin{aligned}
\Lambda \mathcal{D}(\varphi+v) & =\sum_{i, j} a_{i j}(x) D_{i j} v+\sum_{i, j} a_{i j}(x) D_{i j} \varphi+\frac{2}{\varphi+v} \Lambda \\
& \leq \sum_{i, j} a_{i j}(x) \frac{\psi^{\prime \prime}}{\left(\psi^{\prime}\right)^{2}} D_{i} v D_{j} v+\Lambda\left|D^{2} \varphi\right|+\frac{6}{\varepsilon} \Lambda \\
& \leq\left(-b+\beta+\frac{6 \alpha}{\varepsilon}\right) \mathcal{F}
\end{aligned}
$$

whenever $|D v| \geq 1$. Now we choose $b$ and $\delta_{1}$ such that

(1) $b \geq \beta+\frac{6 \alpha}{\varepsilon} \geq 1$

(2) $\delta_{1} b^{2} e^{2 B b} \leq e^{2 B b}-1$

We define $\mathcal{N}=\left\{x \in \Omega, d(x) \leq \delta_{1}\right\}$ where $\delta_{1}$ is sufficiently small. With those choices we have that $|D v|=\psi^{\prime}|D d| \geq 1$ on $\mathcal{N}$ since $|D d|=1$ (we have used (1) and (2)). Clearly, taking into account (1), we deduce $\mathcal{D}(\varphi+v) \leq 0$ on $\mathcal{N}$.

Let $u$ be any solution of the problem $(P)$. We get $u \leq \varphi+v$ on $\partial \mathcal{N} \cap \Omega$ (since $v\left(\delta_{1}\right)=2 B$ ) and on $\mathcal{N} \cap \partial \Omega$ we have also $u \leq \varphi+v$ because $u=f$ on $\partial \Omega$. By the Maximum Principle we deduce that $u \leq \varphi+v$ on $\mathcal{N} \cap \Omega$.

Now choose $\delta<\delta_{0}, \delta_{1}, \delta>0$, such that:

$$
2 B \frac{\log \left(1+|p-x|^{2}\right)}{\log \left(1+\delta_{0}\right)}<\frac{\varepsilon}{3} \quad \text { and }|v(x)|<\frac{\varepsilon}{3}
$$

whenever $|p-x|<\delta$. Then we get $u(x)-u(p)<\varepsilon$ whenever $|p-x|<\delta$.

Consider the function $\bar{\varphi}(x)=f(p)-\frac{\varepsilon}{3}-2 B \frac{\log \left(1+|p-x|^{2}\right)}{\log \left(1+\delta_{0}\right)}$ on $\mathcal{N} \cap \Omega$. Let $U$ be a connected component of $\mathcal{N} \cap \Omega$ where $\bar{\varphi}-v>0$. Let $a>0$ be such that $\left|D^{2} \bar{\varphi}(x)\right|<a$ for any $x \in \mathbf{R}^{2}$. Now set $\tilde{a}_{i j}(x)=\delta_{i j}\left(1+|D \bar{\varphi}-D v|^{2}\right)-D_{i}(\bar{\varphi}-v) D_{j}(\bar{\varphi}-v)$. On $U$ 
we have:

$$
\begin{aligned}
\left(1+|D \bar{\varphi}-D v|^{2}\right) \mathcal{D}(\bar{\varphi}-v) & =-\sum_{i, j} \tilde{a}_{i j} D_{i j} v+\sum_{i, j} \tilde{a}_{i j} D_{i j} \bar{\varphi}+\frac{2\left(1+|D \bar{\varphi}-D v|^{2}\right)}{\bar{\varphi}-v} \\
& =b \sum_{i, j} \tilde{a}_{i j} D_{i} v D_{j} v+\sum_{i, j} \tilde{a}_{i j} D_{i j} \bar{\varphi}+\frac{2\left(1+|D \bar{\varphi}-D v|^{2}\right)}{\bar{\varphi}-v} \\
& \geq b \sum_{i, j} \tilde{a}_{i j} D_{i} v D_{j} v+\sum_{i, j} \tilde{a}_{i j} D_{i j} \bar{\varphi} \\
& \geq b|D v|^{2}-\left|D^{2} \bar{\varphi}\right|\left(1+|D \bar{\varphi}-D v|^{2}\right) \\
& \geq b|D v|^{2}-a\left(1+|D \bar{\varphi}-D v|^{2}\right) \\
& \geq b|D v|^{2}-a\left(2|D \bar{\varphi}|^{2}+2|D v|^{2}+1\right) \\
& \geq(b-2 a)|D v|^{2}-a\left(1+2|D \bar{\varphi}|^{2}\right) .
\end{aligned}
$$

As $|D \bar{\varphi}|$ is bounded (on $\mathbf{R}^{2}$ ), and $|D v| \geq 1$ on $\mathcal{N}$ we will have $\mathcal{D}(\bar{\varphi}-v) \geq 0$ if we choose $b$ big enough in (1). Therefore the Maximum Principle shows that $\bar{\varphi}-v \leq u$ on $U$ for any solution of $(P)$. Thus $\bar{\varphi}-v \leq u$ on $\mathcal{N} \cap \Omega$ for any solution $u$. Therefore for any solution $u$ and for any $p \in \partial \Omega$, and any $x \in \Omega$ such that $|p-x|<\delta$ we have:

$$
|u(x)-u(p)|<\varepsilon
$$

and this shows the modulus of continuity as desired.

COROllaRY 1.8. Consider problem $(P)$ where $\Omega \subset \mathbf{R}^{2}$ is a convex domain in $a$ band and $f$ is bounded, uniformly continuous and non negative on $\partial \Omega$. Then any solution of $(P)$ is uniformly continuous on $\Omega$.

Proof. Let $\varepsilon>0$, then Theorem 1.7 shows the existence of $\delta>0$ such that $|u(p)-u(x)|<\varepsilon$ for any solution $u$ of $(P)$, any $p \in \partial \Omega$ and $x \in \Omega$ such that $|x-p|<\delta$. On the other hand for any $\alpha>0$ the Lemma 1.2 shows that $|D u|$ is bounded on $\Omega_{\alpha}=\{x \in \Omega, d(x, \partial \Omega) \geq \alpha\}$ (as $u$ is bounded on $\Omega$, see Theorem 1.7). This implies that $u$ is uniformly continuous on $\Omega_{\alpha}$ for any $\alpha>0$. Therefore, $u$ is uniformly continuous on $\Omega$.

REMARK 1.9. 1) Let us consider again problem $(P)$ for any domain $\Omega \subset \mathbf{R}^{2}$. Let $p \in \partial \Omega$ and suppose that $\partial \Omega$ is $C^{0}$ convex at $p$ (that is, there exists a straight line $L$ through $p, L \subset \mathbf{R}^{2}$, such that $L \cap \Omega=\emptyset$ ). Then the proof of Theorem 1.7 shows that $p$ admits a barrier.

2) In fact the Theorem 1.7 holds for any dimension. More precisely consider any convex domain of $\mathbf{R}^{n}$ contained in a slab (that is a domain bounded by two parallel hyperplanes of $\mathbf{R}^{n}$ ). Then if $f$ is bounded and uniformly continuous it follows that any solution of $(P)$ admits a modulus of continuity. The proof is the same, working with $n$-dimensional hyperbolic catenoids. Also if we just assume that $\partial \Omega$ is locally convex at $p \in \partial \Omega$ then the proof of Theorem 1.7 shows the existence of a barrier at $p$.

3) Theorem 1.7 does not hold for domain in a proper sector. We will see in the Appendix (Proposition A.1) that there exists a (unique) minimal graph over any proper sector taking zero value boundary data. Moreover, this graph is invariant under homotheties with respect to the vertex of the sector. We deduce that this graph is neither bounded nor uniformly continuous. 
Now we state a straightforward generalization of a Phragmèn-Lindelöf type theorem as founded in 6.1 of [Ro-SE]. The unique difference comparing with [Ro-SE] is that our operator has a term in the form " $h(x) u$ " where $h \leq 0$. Since the proof there applies with minor modifications we will omit it here.

Lemma 1.10. Let $\Omega \subset \mathbf{R}^{n}$ be a domain in a slab. Let $\mathcal{L}(u)=\sum_{i j} a_{i j}(x) D_{i j} u+$ $\sum_{k} b_{k}(x) D_{k} u+h(x) u$ be a linear elliptic operator on $\Omega$ with $h \leq 0$.

Let $\omega \in C^{0}(\bar{\Omega}) \cap C^{2}(\Omega)$ be a function such that:

(1) $\mathcal{L}(\omega) \geq 0$ on $\Omega$.

(2) For each $\delta>0, \delta$ sufficiently small, $\mathcal{L}$ is uniformly elliptic on on $\Omega_{\delta}$. $\Omega_{\delta}=\{x \in \Omega, d(x, \partial \Omega)>\delta\}$, and the coefficients of $\mathcal{L}$ are uniformly bounded

(3) $\omega$ has a modulus of continuity along $\partial \Omega$.

Then if $\omega \leq A$ on $\partial \Omega$ and $\omega \leq B$ on $\Omega$ it follows that $\omega \leq A$ on $\Omega$.

COROLlaRY 1.11. Let $\Omega \subset \mathbf{R}^{n}$ be a convex domain in a slab and let $f$ be $a$ bounded function on $\partial \Omega$, non negative and uniformly continuous. Then problem $(P)$ admits at most one solution.

Proof. Let $u$ and $v$ be two solutions of $(P)$. Observe that $u$ and $v$ are uniformly bounded, see Theorem 1.7. The Theorem 1.7 also shows that $u$ and $v$ have a modulus of continuity. It follows that the function $\omega=u-v$ has also a modulus of continuity. Furthermore we have $\mathcal{L}(\omega)=0$ where $\mathcal{L}$ is the operator defined in the proof of the Proposition 1.4. Note that $\mathcal{L}$ and $\omega$ satisfy the hypothesis of Lemma 1.10 with $A=0$. It follows that $\omega=u-v \leq 0$ on $\Omega$. Setting now $\omega=v-u$ and using the same argument we get that $u=v$ on $\Omega$.

REMARK 1.12. Observe that Theorem 1.7 and Corollary 1.8 and 1.11 hold in the case where $\Omega$ is a proper band. In fact they also hold for more general domains. Namely we can consider domains $\Omega \subset \mathbf{R}^{2}$ such that outside a compact part $K \subset \mathbf{R}^{2}$, $\Omega-K$ is a finite union of convex domains in a band.

\section{Existence and uniqueness of graphs over convex bounded domains.}

Definition 2.1. Let $\Omega \subset \mathbf{R}^{n}$ be a bounded domain such that $\partial \Omega$ is $C^{2}$. We say that $\Omega$ is strictly convex if for every $p \in \partial \Omega$ all the principal curvatures of $\partial \Omega$ at $p$ (with respect to the inward normal orientation) are positive.

THEOREM 2.2. Let $\Omega \subset \mathbf{R}^{n}$ be a bounded strictly convex domain where $\partial \Omega$ is $C^{2, \alpha}$ for some real number $\left.\alpha \in\right] 0,1[$. Let $f: \partial \Omega \rightarrow] 0,+\infty\left[\right.$ be a $C^{2, \alpha}$ function. Then, problem $(P)$ is uniquely solvable. More precisely there exists an unique positive function $u \in C^{2, \alpha}(\bar{\Omega})$ satisfying (Eq. 1-1) and such that $u_{\mid \partial \Omega}=f$.

Proof. Observe that the Remark 1.5 shows the uniqueness of solutions, if any. Hence it suffices to prove the existence part in the statement. Let $a=\inf _{\partial \Omega} f(p)$, we have $a>0$. Let $h: \mathbf{R} \rightarrow \mathbf{R}^{+}$be a $C^{1}$ function such that:

(1) $h(z)=\frac{1}{z+a}$ for $z \geq 0$

(2) $h^{\prime}<0$

(3) $h<\frac{2}{a}$

We wish to use Theorem 1.1, for that we define the following family of Dirichlet 
problems, parametrized by $t \in[0,1]$ :

$$
\left\{\begin{aligned}
\sum_{i, j=1}^{n}\left(\delta_{i j}-\frac{v_{i} v_{j}}{1+|D v|^{2}}\right) v_{i j}+\operatorname{tnh}(v) & =0 \text { on } \Omega \\
v_{\mid \partial \Omega} & =t(f-a)
\end{aligned}\right.
$$

As $f$ admits an euclidean minimal extension (see [Je-Se]) we can suppose that $f \in C^{2, \alpha}(\bar{\Omega})$. Note that for any $t \in[0,1]$, the euclidean mean curvature (with respect to the upward normal orientation) of the graph of any solution $v_{t}$ of $\left(P_{t}\right)$ is negative. It follows that for any $t \in[0,1]$, any solution $v_{t}$ attains its minimum value at the boundary and then it is non negative. Note that this also follows from the Maximum Principle (Proposition 1.4). Therefore any solution $v_{t}$ for any $t$ satisfies:

$$
\begin{aligned}
\sum_{i, j=1}^{n}\left(\delta_{i j}-\frac{v_{i} v_{j}}{1+|D v|^{2}}\right) v_{i j}+t \frac{n}{v+a} & =0 \text { on } \Omega \\
v_{\mid \partial \Omega} & =t(f-a)
\end{aligned}
$$

Hence, for $t=1$ and for any solution $v$ of $\left(P_{1}\right)$ the function $u=v+a$ is a solution of the problem $(P)$. Then to find a $C^{2, \alpha}(\bar{\Omega})$ solution of $(P)$ it suffices to find a $C^{2, \alpha}(\bar{\Omega})$ solution of the problem $\left(P_{1}\right)$. To accomplish this note that the family $\left(P_{t}\right)$ satisfies the hypothesis of Theorem 1.1. It follows that to solve $\left(P_{1}\right)$ (and then to prove Theorem 2.1 ) it suffices to get a real number $A>0$ such that

$$
\sup _{\Omega} v_{t}+\sup _{\Omega}\left|D v_{t}\right|<A
$$

for every $t \in[0,1]$ and every $C^{2, \alpha}(\bar{\Omega})$ solution $v_{t}$ of $\left(P_{t}\right)$. Observe that for each $t$ and any solution $v_{t}$ of $\left(P_{t}\right)$ we have $\mathcal{D}\left(v_{t}+a\right)=(1-t) \frac{n}{v_{t}+a} \geq 0$. Observe also that the graph of $a+t(f-a)$ on $\partial \Omega$ stays below the graph of $f$. Consider now any totally geodesic hyperplane (more shortly geodesic hyperplane) $\Pi$ of $\mathbf{H}^{n+1}$ which stays above the graph of $f$. Namely $\Pi$ is a hemisphere orthogonal to $\mathbf{R}^{n}=\partial_{\infty} \mathbf{H}^{\mathbf{n}+\mathbf{1}}$ above the graph of $f$ (which is compact). Using the Maximum Principle, we deduce that for every $t$ the graph of $v_{t}+a$ stays below $\Pi$ for any $C^{2, \alpha}(\bar{\Omega})$ solution of $\left(P_{t}\right)$. Then using $\Pi$ we get a real number $B>0$ such that $v_{t}<B$ for every $C^{2, \alpha}(\bar{\Omega})$ solution $v_{t}$ of $\left(P_{t}\right)$ and every $t \in[0,1]$.

Now we look for $C^{1}$ apriori estimates. First, note that $v_{t \mid \partial \Omega}$ is $C^{1}$ uniformly bounded. Moreover, observe that the same argument as used at Remark 1.5 shows that for each $t \in[0,1]$ and every solution $v_{t}$ of $\left(P_{t}\right)$, the maximum of $\left|D v_{t}\right|$ is achieved at the boundary of $\Omega$. Therefore it suffices to give uniform $C^{1}$ estimates on the boundary. We begin to give (uniform) lower $C^{1}$ boundary estimates. Let $C^{+}$be the (half) vertical cylinder over $\partial \Omega$. That is we set: $C^{+}=\partial \Omega \times\left[0,+\infty\left[\subset \mathbf{H}^{n+1}\right.\right.$. Let us call $\Gamma_{t}$ the graph of the function $t(f-a)+a$ on $\partial \Omega$. Observe that each $\Gamma_{t}$ is a $C^{2}$ hypersurface of $C^{+}$. Moreover each $\Gamma_{t}$ is contained in the following compact part of $\mathbf{H}^{n+1}: C^{+} \cap\left\{a \leq x_{n+1} \leq \sup _{\partial \Omega} f\right\}$.

Now consider a point $p \in \Gamma_{t}$. Let $P$ be the euclidean tangent hyperplane of $C^{+}$ at $p$. Let $L_{p}$ be the codimension 2 euclidean plane through $p$, tangent to $\Gamma_{t}$. For any $\theta \leq 0$, let us define $P_{\theta}$ to be the hyperplane $P$ rotated by $\theta$ with respect to $L_{p}$. That is, we require that $P_{\theta} \cap C^{+}$stays below the euclidean hyperplane orthogonal to $C^{+}$at $p$ containing $L_{p}$ and the angle between $P_{\theta}$ and the vertical hyperplane $P$ is $\theta$. Observe that when $\theta=0$ we have $P_{0}=P$ and $C^{+} \cap P$ is the vertical line through $p$. 
Clearly when $|\theta|$ is small enough $C^{+} \cap P_{\theta}$ is a hypersurface of $C^{+}$which is a vertical graph, over a part of $\partial \Omega$, staying below $\Gamma_{t}$. Now as all the hypersurfaces $\Gamma_{t}, t \in[0,1]$ are uniformly bounded up to the $C^{2}$ topology, and they stay in a compact part of $\mathbf{H}^{n+1}$, there exists an uniform $\theta<0$ such that for each $t \in[0,1]$ and each $p \in \Gamma_{t}$ we have that $C^{+} \cap P_{\theta}$ stays below $\Gamma_{t}$ and $\left(C^{+} \cap P_{\theta}\right) \cap \Gamma_{t}=\{p\}$. Now we show that for any $t \in[0,1]$, for any solution $v_{t}$ of $\left(P_{t}\right)$ and any point $p \in \Gamma_{t}$, the graph of $v_{t}+a$ stays above the compact part of $P_{\theta}$ bounded by $C^{+}$. Then this will give a lower $C^{1}$ estimate of $v_{t}$ at $p$, independent of $p, t$ and $v_{t}$. Indeed, choose $t, \Gamma_{t}$ and $p \in \Gamma_{t}$ as above and call $u_{t, p}$ the $C^{2}$ function on $\bar{\Omega}$ such that its graph is precisely the euclidean hyperplane $P_{\theta}$. Observe that we have $\left(v_{t}+a\right)_{\mid \partial \Omega} \geq\left(u_{t, p}\right)_{\mid \partial \Omega}$. Suppose it is not true that $u_{t, p} \leq v_{t}+a$. Then there exists a real number $b>0$ such that $u_{t, p} \leq v_{t}+a+b$ with equality occuring at an interior point of $\Omega$. But this gives a contradiction with the Maximum Principle since on the part of $\Omega$ where $u_{t, p}>0$ we have:

$$
\mathcal{D}\left(u_{t, p}\right)=\frac{n}{u_{t, p}} \geq \frac{n(1-t)}{v_{t}+a+b}=\mathcal{D}\left(v_{t}+a+b\right) .
$$

Now we look for upper $C^{1}$ apriori boundary estimates. We begin as before but now we consider the positive rotated hyperplanes $P_{\alpha}, \alpha>0$. That is $P_{\alpha} \cap C^{+}$stays above $\Gamma_{t}$. The same argument shows there exists $\alpha>0$ such that the latter is true for each $t \in[0,1]$, for every solution $v_{t}$ of $\left(P_{t}\right)$ and each $p \in \Gamma_{t}$. Unfortunately, we cannot apply the Maximum Principe to conclude the proof (because there is no contradiction to the fact that $P_{\alpha}$ stays over the graph of $v_{t}+a$ with a tangent point of contact). But observe that this situation is not possible substituting (euclidean) hyperplanes by (hyperbolic) geodesic hyperplanes. Observe also that if $\alpha>0$ is small enough, the euclidean hyperplane $P_{\alpha}$ is almost vertical. Therefore, the (unique) geodesic hyperplane $S_{\alpha}$ of $\mathbf{H}^{n+1}$ through $p \in \Gamma_{t}$ tangent at $P_{\alpha}$ is $C^{2}$ close to $P_{\alpha}$ in a compact part of $\mathbf{H}^{n+1}$. Now choose a compact part $K$ of $\mathbf{H}^{n+1}$ including in its interior the set $C^{+} \cap\left\{a \leq x_{n+1} \leq \sup _{\partial \Omega} f\right\}$. That is $S_{\alpha} \cap C^{+}$is $C^{2}$ close of $P_{\alpha} \cap C^{+}$ in $K$. We infer that if $\alpha>0$ is small enough each intersection $S_{\alpha} \cap C^{+}$stays over $\Gamma_{t}$, for each $t \in[0,1]$, each solution $v_{t}$ of $\left(P_{t}\right)$ and each $p \in \Gamma_{t}$. Using the Maximum Principle as in the proof of the lower $C^{1}$ apriori estimates, we get that the graph of $S_{\alpha}$ over $\Omega$ stays over the graph of $v_{t}+a$, with intersection at the boundary point $p$. This gives upper $C^{1}$ boundary estimates and concludes the proof.

Theorem 2.3. Let $\Omega \subset \mathbf{R}^{2}$ be a compact $C^{0}$ convex domain and let $f: \partial \Omega \rightarrow$ $[0,+\infty[$ be a continuous function. Then problem $(P)$ admits an unique solution.

Proof. When $f \equiv 0$ the result follows from Theorems 3-4. We will proceed the proof for $f \not \equiv 0$. In view of Remark 1.5 it suffices to prove the existence of a solution. Let $F: \bar{\Omega} \rightarrow[0,+\infty$ [ be the (unique) euclidean minimal extension of $f$ over $\Omega$. Note that the usual maximum principle for minimal surface of $\mathbf{R}^{3}$ shows that $F(x)>0$ (as $F_{\mid \partial \Omega}=f \geq 0$ ) for any $x \in \Omega$. This can be shown also using the Maximum Principle in Proposition 1.4. Consider now a compact exhaustion $\left(K_{n}\right), n \in \mathbf{N}$, of $\bar{\Omega}$ such that for each $n, K_{n} \subset \Omega$ is a compact part of $\Omega$, homeomorphic to a closed disk, and $\partial K_{n}$ is a $C^{3}$ strictly convex Jordan curve. Let us call $\left.u_{n}: K_{n} \rightarrow\right] 0,+\infty[$ the unique hyperbolic minimal extension of $F_{\mid \partial K_{n}}$ given by the Theorem 2.2. Hence $\mathcal{D}\left(u_{n}\right)=0$ in $\operatorname{Int}\left(K_{n}\right)$ and $u_{n}=F$ on $\partial K_{n}$. Moreover the Maximum Principle shows that $u_{n}>F$ on $\operatorname{Int}\left(K_{n}\right)$ for each $n$. Let $\Pi$ be any geodesic plane above the graph of $f$ on $\partial \Omega$. Namely $\Pi$ is a half-sphere orthogonal to $\partial_{\infty} \mathbf{H}^{3}=\mathbf{R}^{2}$ containing the graph of $f$ in its "interior". Now choose $K_{n_{0}}$ where $n_{0} \in \mathbf{N}$ is a fixed integer and consider the functions 
$u_{n}$ for $n>n_{0}$. The above observations show the existence of two positive numbers $a, b$ such that $0<a<u_{n}<b$ on $K_{n_{0}}$ for any $n>n_{0}$. The Compactness Theorem 1.3 gives rise to a subsequence of $\left(u_{n}\right)$ converging in the $C^{2}$-topology to a positive $C^{2}$ function $u$ on $\Omega$ satisfying (Eq. 1-1). By abuse of notation we continue to call $\left(u_{n}\right)$ this subsequence. It remains to prove that $u$ is continuous in $\bar{\Omega}$ and $u_{\mid \partial \Omega}=f$.

Observe that by construction we have $F \leq u$ on $\Omega$. Furthermore, the graph of $u$ stays below the geodesic plane $\Pi$ as this is true for each $u_{n}$. Let $B>0$ be the maximum height of $\Pi$, then $u<B$ on $\Omega$. For each $n$ let $U_{n}: \bar{\Omega} \rightarrow[0,+\infty[$ be the continuous function defined by

$$
U_{n}(x)=\left\{\begin{array}{llc}
u_{n}(x) & \text { for } & x \in K_{n} \\
F(x) & \text { for } & x \in \bar{\Omega}-K_{n} .
\end{array}\right.
$$

Clearly $U_{n}$ converges to $u$ on $\Omega$. Let $p \in \partial \Omega$ be any fixed point, as $\partial \Omega$ is convex $p$ admits a superior barrier, see Remark 1.9. Therefore there exists an open neighborhood $\mathcal{N}$ of $p$ in $\mathbf{R}^{2}$ and a sequence of functions $\omega_{k}^{+}$in $C^{2}(\mathcal{N} \cap \Omega) \cap C^{0}(\overline{\mathcal{N} \cap \Omega)}$ satisfying conditions $(i),(i i)$ and $(i i i)$ of Definition 1.6, where $M$ is substituted by $B$. The Maximum Principle implies that $\omega_{k}^{+} \geq F$ on $(\overline{\mathcal{N} \cap \Omega)})$ for each $k$. This proves that $\omega_{k}^{+} \geq U_{n}$ on $\mathcal{N} \cap \partial K_{n}$ for each $k$ and $n$. Observe also that $\omega_{k}^{+} \geq B>U_{n}$ on $\partial \mathcal{N} \cap K_{n}$. The latter and the Maximum Principle yield $\omega_{k}^{+} \geq U_{n}$ on $\left(\overline{\mathcal{N} \cap K_{n}}\right)$. Finally we get $\omega_{k}^{+} \geq U_{n}$ on $(\overline{\mathcal{N} \cap \Omega})$ for any $k$ and $n$. Let any $x \in \mathcal{N} \cap \Omega$, we have $F(x) \leq u(x) \leq w_{k}^{+}(x)$ and then $F(x)-f(p) \leq u(x)-f(p) \leq w_{k}^{+}(x)-f(p)$. When $x$ converges to $p$ and $k \rightarrow+\infty$ we get that $u(x)$ converges to $f(p)$. That is $u$ is continuous up to $p$ and $u(p)=f(p)$.

The next result shows that the vertical graphs constructed in Theorem 2.3 are unique in the class of minimal surface with the same boundary.

TheOREM 2.4. Let $\Omega \subset \mathbf{R}^{n}$ be a strictly $C^{2}$ convex domain and let $M \subset \mathbf{H}^{n+1}$ be a compact (hyperbolic) minimal hypersurface continuous up to the boundary. Suppose that $\Gamma=\partial M$ is a continuous vertical graph over $\partial \Omega$. Then the whole hypersurface $M$ is a $C^{2}$ vertical graph over $\Omega$. Furthermore $M$ is the unique connected and compact immersed minimal hypersurface whose boundary is $\Gamma$. The same statement hold when $n=2$ and $\partial \Omega$ is only supposed to be a $C^{0}$ convex domain.

Proof. Let $\left.C^{+}=\partial \Omega \times\right] 0,+\infty\left[\subset \mathbf{H}^{n+1}\right.$ be the (half) cylinder over $\partial \Omega$. Then $\Gamma=\partial M \subset C^{+}$and by the Maximum Principle the whole hypersurface $M$ stays inside the solid cylinder bounded by $C^{+}$, that is in $\left.\bar{\Omega} \times\right] 0,+\infty[$. To see this observe that the vertical (euclidean) hyperplanes are geodesic hyperplanes, and then are minimal hypersurfaces of $\mathbf{H}^{n+1}$. For the same reason, $M$ cannot have any interior point on the cylinder $C^{+}$. Let $x \in \Omega$ be any point. Consider the $\lambda$-homotheties, $h_{\lambda}$, with respect to $x$, with $\lambda>0$ and recall that they are hyperbolic isometries. More precisely we have $h_{\lambda}(X)=\lambda(X-x)+x$ for any $X \in \mathbf{H}^{n+1}$. As $\partial \Omega$ is strictly convex, $\Gamma$ is a radial graph with respect to $x$, that is $h_{\lambda}(\Gamma) \cap \Gamma=\emptyset$ for any positive $\lambda \neq 1$. We have also that $h_{\lambda}(\Gamma) \cap M=\emptyset$ for any $\lambda>1$ since $M$ stays in the convex closure of $C^{+}$.

Suppose now that $M$ is not a radial graph with respect to $x$. Hence, there exists $\lambda>1$ such that $h_{\lambda}(M) \cap M \neq \emptyset$. But the last implies the existence of $\lambda_{0} \geq \lambda$ such that $h_{\lambda_{0}}(M)$ and $M$ are tangent at some interior point and $h_{\lambda_{0}}(M)$ stays "above" $M$ in the sense of radial graph (with respect to $x$ ). Now the Maximum Principle applies to induce that $h_{\lambda_{0}}(M)=M$ which is absurd since $\lambda_{0}>1$ and then $h_{\lambda_{0}}(\partial M) \cap \partial M=\emptyset$. 
Therefore we conclude that the whole hypersurface is a radial graph with respect to $x$. In particular, the intersection between $M$ and the vertical line through $x$ is reduced to a single point. Since the previous argument holds for any $x \in \Omega$, we deduce that $M$ is a vertical graph over $\bar{\Omega}$. It remains to prove that this vertical graph is $C^{2}$. That is $M$ no has interior point with vertical tangent space. Suppose there exists an interior point $X \in M$ such that the tangent space of $M$ at $X$ is vertical. Let us call $x \in \Omega$ the orthogonal projection of $X$ on $\mathbf{R}^{n}=\partial_{\infty} \mathbf{H}^{n+1}$. We know that $M$ is a radial graph with respect to $x$. Consider the following family $\Pi_{t}, t>0$, of geodesic hyperplanes of $\mathbf{H}^{n}$ : for each $t>0, \Pi_{t}$ is the hemisphere centered to $x$ with (euclidean) radius $t$. When $t$ is big enough $\Pi_{t}$ no has intersection with $M$ and $M$ stays below $\Pi_{t}$. When $t$ decreases to 0 we get a first contact point between $M$ and a hemisphere $\Pi_{t_{0}}$. Now we use the Alexandrov reflection to conclude. Namely for $t<t_{0}$, we call $M_{t}^{+}$the part of $M$ being above $\Pi_{t}$ and $M_{t}^{-}$the part being below $\Pi_{t}$. Furthermore we call $M_{t}^{*}$ the reflected of $M_{t}^{+}$with respect to $\Pi_{t}$. Recall that reflections with respect to hemispheres orthogonal to $\partial_{\infty} \mathbf{H}^{n}$ are hyperbolic isometries. Hence $M_{t}^{*}$ is again a hyperbolic minimal hypersurface. As $M$ is a radial graph (with respect to $x$ ) the intersection between $M_{t}^{-}$and $M_{t}^{*}$ only occurs on their common boundary, that is on $\Pi_{t}$. It follows from the boundary Maximum Principle (see Remark $1.5(4)$ ) that $M_{t}^{-}$ and $M_{t}^{*}$ cannot be tangent on their common boundary. But when we choose $\Pi_{t}$ to be the hemisphere containing $X$ we get:

$$
X \in \partial M_{t}^{*} \cap \partial M_{t}^{-}, M_{t}^{-} \cap M_{t}^{*}=\partial M_{t}^{-}=\partial M_{t}^{*} .
$$

Furthermore the tangent spaces of $M_{t}^{-}$and $M_{t}^{*}$ at $X$ are the same since the tangent space of $M$ at $\mathrm{X}$ is vertical. We deduce from the boundary Maximum Principle that $M_{t}^{-}=M_{t}^{*}$, that is $M$ is symmetric with respect to $\Pi_{t}$. But this is absurd since $\partial M$ is not symmetric.

The uniqueness of $M$ comes from the uniqueness of vertical graph with fixed continuous boundary data, see the Remark 1.5.

Clearly the whole construction is valid when $n=2$ and $\partial \Omega$ is only supposed $C^{0}$ convex.

REMARK 2.5. Note that Theorems 2.3 and 2.4 do not hold any longer if $\Omega$ is neither convex neither connected. Indeed, consider two positive real numbers $0<$ $r<1<R$. Let $U \subset \mathbf{H}^{3}$ be the region delimited by the two hemispheres (geodesic hyperplanes) centered at 0 whose radius is respectively $r$ and $R$. It is known that for $r, R$ properly chosen, the region $U$ contains a family $\mathcal{F}$ of hyperbolic catenoids whose axis of rotation is the vertical axis $\left\{x_{1}=x_{2}=0\right\}$. Furthermore each catenoid of this family is symmetric with respect to the hemisphere $\Pi$ centered at 0 whose radius is 1 . For every point $P \in \Pi, P \neq(0,0,1)$, there is a catenoid of $\mathcal{F}$ intersecting orthogonally $\Pi$ at $P$.

Now we choose $\Omega \subset \mathbf{R}^{2}=\partial_{\infty} \mathbf{H}^{3}$ to be an annular domain bounded by two circles centered at 0 with radius $r<r_{1}<1<r_{2}<R$. If $r_{1}$ and $r_{2}$ are closed enough of 1 , one of the catenoid of $\mathcal{F}$ contains a part $S$ bounded by two horizontal circles, one of those circles is a graph over the circle of radius $r_{1}$ and the other is a graph over the circle of radius $r_{2}$. But $S$ is not a graph, thus Theorem 2.4 does not hold for the domain $\Omega$.

Now we choose $r_{1}<r<R<r_{2}$. Then the Dirichlet problem $(P)$ with $f \equiv 0$ has no solution. Indeed, suppose $M \subset \mathbf{H}^{3}$ is a minimal surface, which is a graph over $\Omega$ and such that $\partial_{\infty} M=\partial \Omega$. Then by an argument of continuity, we could 
get a catenoid $C$ of $\mathcal{F}$ tangent to $M$ and staying below $M$. But the latter yields a contradiction with the Maximum Principle. We conclude that Theorem 2.3 does not hold for the domain $\Omega$.

3. Perron process, elliptic, hyperbolic and parabolic surfaces. Now we describe the Perron process (see [Co-Hi], chapter IV, §4), for minimal surfaces of $\mathbf{H}^{3}$. The Perron process was applied in the theory of minimal surfaces in $\mathbf{R}^{3}$. See, for instance, [Je-Se, 1], [Je-Se, 2] and [SE-To, 1].

Definition 3.1. Consider problem $(P)$ where $\Omega$ is any domain of $\mathbf{R}^{2}$ and $f$ is any non negative continuous function on $\partial \Omega$.

1) Let $u: \bar{\Omega} \rightarrow[0,+\infty$ [ be a continuous function. Let $U \subset \Omega$ be a closed round disk. It follows from Theorem 2.3 that $u_{\mid \partial U}$ has an unique minimal extension $\tilde{u}$ on $U$, continuous up to $\partial U$. We then define the continuous function $M_{U}(u)$ on $\bar{\Omega}$ by:

$$
M_{U}(u)(x)= \begin{cases}u(x) & \text { if } x \in \bar{\Omega}-U \\ \tilde{u}(x) & \text { if } x \in U\end{cases}
$$

2) Let $u: \bar{\Omega} \rightarrow[0,+\infty[$ be a continuous function. We say that $u$ is a subsolution (resp. supersolution) of $(P)$ if:

(i) $u_{\mid \partial \Omega} \leq f$ (resp. $\left.u_{\mid \partial \Omega} \geq f\right)$.

(ii) For any closed round disk $U \subset \Omega$ we have $u \leq M_{U}(u)$ (resp. $u \geq M_{U}(u)$ ).

REMARK 3.2. We now give some classical facts about subsolutions and supersolutions, see [Co-Hi].

1) It is easily seen that if $u$ is $C^{2}$ on $\Omega$ and if $u>0$, the condition (ii) in Definition 3.1 is equivalent to $\mathcal{D}(u) \geq 0$ for subsolution or $\mathcal{D}(u) \leq 0$ for supersolution.

2) As usual if $u$ and $v$ are two subsolutions (resp. supersolutions) of $(P)$ then $\sup (u, v)$ (resp. $\inf (u, v))$ again is a subsolution (resp. supersolution).

3) Also if $u$ is a subsolution (resp. supersolution) and $U \subset \Omega$ is a closed round disk then $M_{U}(u)$ is again a subsolution (resp. supersolution).

4) Let $\Omega \subset \mathbf{R}^{2}$ be a bounded domain and let $u, v: \bar{\Omega} \rightarrow[0,+\infty$ [ be two continuous functions such that $M_{U}(u) \geq u$ and $M_{U}(v) \leq v$ for any closed round disk $U \subset$ $\Omega$. Suppose that $u_{\mid \partial \Omega} \leq v_{\mid \partial \Omega}$, then we have $u \leq v$ on $\Omega$. Roughly speaking, a supersolution is greater than a subsolution.

EXAMPLE 3.3. 1) For any domain $\Omega \subset \mathbf{R}^{2}$, if $f$ is any continuous positive function on $\partial \Omega$, then the vanishing function $u \equiv 0$ on $\bar{\Omega}$ is a subsolution for $(P)$. Observe that for every $x \in \Omega$ there exists a subsolution $u$ of $(P)$ with $u(x)>0$. Indeed let $\Pi$ be any hemisphere centered at $x$ such that $\partial_{\infty} \Pi \subset \Omega$. Then $\Pi$ is the graph of a continuous function $v$ defined on a closed round disk $U \subset \Omega$. Then set $u=v$ on $U$ and $u=0$ on $\bar{\Omega}-U$. One easily verify that the function $u$ is a subsolution.

2) Choose $\Omega$ to be a proper sector of $\mathbf{R}^{2}$. That is a region of $\mathbf{R}^{2}$ bounded by two rays issue from the same point $x \in \mathbf{R}^{2}$ and making an angle $\left.\theta \in\right] 0, \pi[$ at $x$. In the Appendix, Proposition A.1, it is shown that there exists a solution $u_{\theta}$ for problem $(P)$ where $f \equiv 0$ is the null function. In fact the graph of $u_{\theta}$ is invariant by any homotheties $h_{x, \lambda}$ with center $x$ and ratio $\lambda>0$. More precisely, we have $u_{\theta}\left(h_{x, \lambda}(p)\right)=\lambda \cdot u_{\theta}(p)$ for any $p \in \Omega$. We deduce that $u_{\theta}$ is a supersolution for $(P)$ (with $f \equiv 0$ ). In fact, we 
will prove in $\S 4$ (Corollary 4.6) that $u_{\theta}$ is the unique solution of $(P)$.

In the same way let $\Omega$ be a band of $\mathbf{R}^{2}$. Up to an isometry of $\mathbf{H}^{3}$, we can suppose that $\Omega=\left\{(x, y) \in \mathbf{R}^{2}, 0<y<1\right\}$. Consider problem $(P)$ where $f \equiv 0$ is again the null function. Also in the Appendix, Proposition A.2, it is proved that problem $(P)$ has a solution $u_{B}$. Again we deduce that $u_{B}$ is a supersolution for $(P)$. In fact the graph of $u_{B}$ is invariant under any horizontal translation fixing the band $\Omega$. That is we have $u_{B}(p+(x, 0))=u_{B}(p)$ for any $p \in \Omega$ and any $x \in \mathbf{R}$. We will prove that $u_{B}$ is the unique solution of $(P)$ in $\S 4$ (see Corollary 4.2).

3) Let $\Omega \subset \mathbf{R}^{2}$ be an unbounded domain in a band. Let $f$ be any non negative and bounded continuous function on $\partial \Omega$. Then using again the hyperbolic catenoids and the horizontal translations parallel to the band, in the same way as in the proof of Theorem 1.7, we can show that any solution for the problem $(P)$ is bounded. Therefore, using a well chosen band $B$ containing $\Omega$, such that the solution $u_{B}$ (see item 2 above) envolves $f$, that is such that $u_{B \mid \partial \Omega} \geq f$, we get a supersolution for problem $(P)$, invariant under any horizontal translations parallel to the band. Namely, the supersolution is $u_{B \mid \bar{\Omega}}$.

4) Now let $\Omega \subset \mathbf{R}^{2}$ be any bounded domain and let $f: \partial \Omega \rightarrow[0,+\infty[$ be any continuous function. It is easy to construct a supersolution for problem $(P)$. Indeed, let $C \subset \mathbf{H}^{3}$ be a hyperbolic catenoid such that its orthogonal projection, $C_{0}$, on $\mathbf{R}^{2}$ has a non empty intersection with $\Omega$. Let $x \in \Omega$ be an interior point of $C_{0}$. Consider the homotheties $h_{\lambda, x}$ with respect to $x, \lambda>0$. Clearly, for $\lambda>1$ big enough, a piece of $h_{\lambda, x}(C)$ is a vertical graph $v$ over a domain containing $\bar{\Omega}$ and $v_{\mid \partial \Omega}>\sup (f)$. That is, $v$ is a supersolution for $(P)$.

Theorem 3.4. (Perron process) Let $\Omega \subset \mathbf{R}^{2}$ be a domain and let $f: \partial \Omega \rightarrow$ $[0,+\infty[$ be a continuous fonction. Suppose that problem $(P)$ has a supersolution $\phi$. Set $\mathcal{S}_{\phi}=\{u$, subsolution of $(P), u \leq \phi\}$. We define for each $x \in \bar{\Omega}$

$$
v(x)=\sup _{u \in \mathcal{S}_{\phi}} u(x) \text {. }
$$

Then the function $v$ is $C^{2}$ on $\Omega$ and satisfies (Eq. 1-1). holds:

Furthermore, consider $p \in \partial \Omega$ and suppose that either one of the following cases

(i) $\partial \Omega$ is $C^{0}$-convex at $p$.

(ii) $p$ has a barrier and $f \geq \alpha>0$ on $\partial \Omega$.

(iii) $p$ has a barrier and $f(p)=0$.

Then $v$ is continuous up to $p$ and $v(p)=f(p)$. In particular if $\Omega$ is $C^{0}$ convex, the function $v$ is continuous up to $\partial \Omega$.

Proof. First observe that $\mathcal{S}_{\phi} \neq \emptyset$ since the vanishing function belongs to $\mathcal{S}_{\phi}$. By construction we have $v \leq \phi$ on $\bar{\Omega}$. Let $u \in \mathcal{S}_{\phi}$ and let $U \subset \Omega$ be a closed round disk. Note that $M_{U}(u) \in \mathcal{S}_{\phi}$. Indeed we know that $M_{U}(u)$ is a subsolution. Furthermore the graphs of $M_{U}(u)$ and $M_{U}(\phi)$ over $U$ are two minimal surfaces such that the graph of $M_{U}(u)=u$ on $\partial U$ is below the graph of $M_{U}(\phi)=\phi$ over $\partial U$. The Maximum Principles induces that $M_{U}(u) \leq M_{U}(\phi)$. But by the very definition of supersolution we have $M_{U}(\phi) \leq \phi$ and then $M_{U}(u) \leq \phi$.

Now we show that $v$ is $C^{2}$ on $\Omega$ and satisfies (Eq. 1-1). Let $x \in \Omega$ be any point. Consider a sequence $\left(u_{n}\right)$ in $\mathcal{S}_{\phi}$ such that $u_{n}(x) \rightarrow v(x)$ when $n \rightarrow+\infty$. Let $U \subset \Omega$ be any closed round disk centered at $x$. We can assume that for each $n$ and each $y \in \partial U$ we 
have $u_{n}(y)>0$. Define for each $n$ the function $v_{n}(p)=\sup \left\{u_{1}(p), \ldots, u_{n}(p)\right\}, p \in \bar{\Omega}$. By construction we have also $M_{U}\left(v_{n}\right)(x) \rightarrow v(x)$ when $n \rightarrow+\infty$. Set $V_{n}=M_{U}\left(v_{n}\right)$. Therefore $V_{n}$ is an increasing sequence of functions bounded above by $\phi$, satisfying (Eq. 1-1) on $U$. We deduce the existence of two real numbers $0<a<b$ such that $a<V_{n}(p)<b$ for each $n$ and every $p \in U$. Now we get with Theorem 1.3 that a subsequence of $\left(V_{n}\right)$ converges to a $C^{2}$ function $V$ on $\operatorname{Int}(U)$ satisfying (Eq. 1-1). Let us call again $\left(V_{n}\right)$ this subsequence by an abuse of notation. By construction we have $V(x)=v(x)$. It remains to prove that $V(p)=v(p)$ for any $p \in \operatorname{Int}(U)$. Let $y \in$ $\operatorname{Int}(U)$ be a fixed point. Let $\left(\bar{u}_{n}\right)$ in $\mathcal{S}_{\phi}$ such that $\bar{u}_{n}(y) \rightarrow v(y)$ when $n \rightarrow+\infty$. Set $\bar{v}_{n}=\sup \left(V_{n}, \bar{u}_{n}\right)$. As above we can assume that $\left(\bar{v}_{n}\right)$ is an increasing sequence. Set $\bar{V}_{n}=M_{U}\left(\bar{v}_{n}\right)$, then there exist two real numbers $0<\bar{a}<\bar{b}$ such that $\bar{a}<\bar{V}_{n}(p)<\bar{b}$ for any $p \in U$ and any $n$. It follows from the Compactness Theorem (Theorem 1.3) that a subsequence of $\left(\bar{V}_{n}\right)$ converges on $U$ in the $C^{2}$ topology to a $C^{2}$ function $\bar{V}$ on $U$ satisfying (Eq. 1-1). We have $\bar{u}_{n}(y) \leq \bar{V}_{n}(y) \leq v(y)$, we infer that $\bar{V}(y)=v(y)$. Also by construction we have $V_{n} \leq \bar{v}_{n} \leq \bar{V}_{n}$, the last inequality holds since $\bar{v}_{n}$ is a subsolution of $(P)$. Therefore we get $V \leq \bar{V}$, from which we deduce $\bar{V}(x)=v(x)$ (because $\bar{V} \leq v)$. Now, observe that $\bar{V}$ and $V$ are $C^{2}$ functions on $\operatorname{Int}(U)$ satisfying (Eq. 1-1) such that $V \leq \bar{V}$ with equality at an interior point $x$. The Maximum Principle applies here to deduce that $V=\bar{V}$ on $\operatorname{Int}(U)$. In particular $V(y)=v(y)$, as this is true for any $y \in \operatorname{Int}(U)$ we conclude that $v=V$ on $\operatorname{Int}(U)$. This shows the first part of the theorem.

Let $p \in \partial \Omega$ be a boundary point. Observe that for each of the three cases $p$ admits a superior barrier. Therefore, there exist a neighborhood $\mathcal{N}$ of $p$ and a sequence of functions $\omega_{k}^{+}$, as in Definition 1.6 where $M>0$ is chosen so that $M>\sup _{\partial \mathcal{N} \cap \Omega}(\phi(x))$. For each $k$ and any $u \in \mathcal{S}_{\phi}$ we have $\omega_{k}^{+}(x) \geq u(x)$ for every $x \in \partial(\mathcal{N} \cap \Omega)$. We infer that $\omega_{k}^{+} \geq v$ on $\mathcal{N} \cap \Omega$ for each $k$, see Remark 3.2 (4).

Now, suppose that case $(i)$ occurs. We define for $x \in \bar{\Omega}$,

$$
h_{k}(x)=f(p)-\frac{\varepsilon_{k}}{3}-\log \left(1+|p-x|^{2}\right)-\log \left(1+\frac{e^{2 b}-1}{\delta_{k}} d(x)\right),
$$

where $\left(\varepsilon_{k}\right)$ and $\left(\delta_{k}\right)$ are monotonous sequences of positive real numbers such that: $\varepsilon_{k} \rightarrow 0, \delta_{k} \rightarrow 0$ when $k \rightarrow+\infty, \phi(x)>f(p)-\frac{\varepsilon_{k}}{3}$ if $|p-x|<\delta_{k}$ and $h_{k}(x)<0$ if $|p-x| \geq \delta_{k}$. Furthermore, we want that $\delta_{k} e^{b} \leq e^{b}-1$ and $\mathcal{D}\left(h_{k}\right) \geq 0$ in the part where $h_{k}(x)>0$ and $d(x)<\delta_{k}$, see the proof of Theorem 1.7. Therefore, we can choose $\omega_{k}^{-}(x)=\max \left(0, h_{k}(x)\right)$ for $x \in \bar{\Omega}$. In fact, note that for every $k \in \mathbf{N}$, we have that $\omega_{k}^{-}(x)<\phi(x)$ on $\bar{\Omega}$ and $M_{U}\left(\omega_{k}^{-}\right) \geq \omega_{k}^{-}$, that is $\omega_{k}^{-} \in \mathcal{S}_{\phi}$ for every $k$. Therefore $\omega_{k}^{-}<v$. We infer that

$$
\omega_{k}^{-}(x)-f(p) \leq v(x)-f(p) \leq \omega_{k}^{+}(x)-f(p)
$$

for every $k$ and every $x \in \mathcal{N} \cap \Omega$. When $x$ converges to $p$ and $k$ converges to $+\infty$ we get that $v(x)$ converges to $f(p)$ as desired.

Suppose now that case $(i i)$ holds. In particular $p$ has an inferior barrier. Let $\mathcal{N}$ be a neighborhood of $p$ and $\omega_{k}^{-}$a sequence of functions as in Definition 1.6, where $n>0$ is choosen so that $1 / n<\alpha$. Set $\beta_{k}=\sup _{\partial \mathcal{N} \cap \Omega} \omega_{k}^{-}(x)$, we have $\beta_{k}<\alpha$ since $\omega_{k}^{-}<1 / n$ on $\partial \mathcal{N} \cap \Omega$. We define $u_{k}$ on $\bar{\Omega}$ setting $u_{k}(x)=\max \left(\omega_{k}^{-}(x), \beta_{k}\right)$ for $x \in \overline{\mathcal{N} \cap \Omega}$ and $u_{k}(x)=\beta_{k}$ for $x \in \bar{\Omega}-\mathcal{N}$. Observe that $u_{k}$ is a continuous function on $\bar{\Omega}$ and is a subsolution for problem $(P)$. We infer that $u_{k} \leq v \leq \omega_{k}^{+}$on $\mathcal{N} \cap \Omega$ for every $k \in \mathbf{N}$. We deduce that when $x$ converges to $p$ and $k$ converges to $+\infty$, then $v(x)$ converges 
to $f(p)$.

Finally assume that case (iii) holds. Consequently, we get $0 \leq v \leq \omega_{k}^{+}$on $\mathcal{N} \cap \Omega$. We conclude that $v(x)$ converges to $0=f(0)$ when $x$ converges to $p$.

The following result is a straightforward consequence of Theorem 3.4.

Corollary 3.5. Let $\Omega \subset \mathbf{R}^{2}$ be an unbounded domain and let $f$ be any non negative continuous function on $\partial \Omega$. Suppose there exists a supersolution $\phi$ for problem $(P)$. Let $\Gamma \subset \partial \Omega$ be a convex arc, if any, with respect to the interior transversal orientation of $\partial \Omega$. Then the solution $v$ of Perron, relative to $\phi$, given in Theorem 3.4 is continuous up to $\Gamma$ and $v(p)=f(p)$ for any $p \in \Gamma$.

REMARK 3.6. 1) The same holds for any bounded domain. Namely consider problem $(P)$ where $\Omega$ is a bounded domain and $f$ is any non negative function on $\partial \Omega$. Note that problem $(P)$ admits always a supersolution $\phi$, see Example 3.3 (4). Let $\Gamma \subset \partial \Omega$ be a convex arc, if any. Therefore the solution of Perron $v$ relative to $\phi$ is continuous up to $\Gamma$ and $v(p)=f(p)$ for any $p \in \Gamma$.

2) We remark that in order to proof Theorem 3.4 (Perron process), we just need to applied Theorem 2.3 when $\partial \Omega$ is a round circle.

Now we show that for each type of positive isometry of $\mathbf{H}^{3}$ (namely elliptic, hyperbolic and parabolic isometries), there exists a complete minimal vertical graph in $\mathbf{H}^{3}$ invariant by the action of a discrete group of such isometries of $\mathbf{H}^{3}$.

Proposition 3.7. (Elliptic Surfaces) Let $n \in \mathbf{N}, n>0$, be any integer and set $\theta_{n}=\frac{2 \pi}{n}$. Let $R_{n}$ be the rotation with respect to the $x_{3}$-axis whose argument is $\theta_{n}$. Then there exist a complete and a non complete minimal surfaces in $\mathbf{H}^{3}$ invariant by the rotation $R_{n}$.

Proof. Recall that $R_{n}(p)=e^{i \theta_{n}} . p$ for any $p \in \mathbf{R}^{2}$. Let $\Gamma_{n} \subset \mathbf{R}^{2}$ be a regular polygon centered at the origin 0 , with $n$ sides. Let $\Omega \subset \mathbf{R}^{2}$ be the domain whose boundary is $\Gamma_{n}$. Consider $f: \Gamma_{n} \rightarrow[0,+\infty$ [ any continuous function invariant by $R_{n}$. That is $f\left(R_{n}(p)\right)=f(p)$ for every $p \in \Gamma_{n}$. We know that problem $(P)$ has a supersolution $\phi$ (see Example $3.3(4)$ ). Therefore, as $\partial \Omega$ is $C^{0}$ convex we conclude with Remark 3.6 that the solution of Perron $v$ (relative to $\phi$ ) given in Theorem 3.4 is continuous up to the boundary and $v(p)=f(p)$ for any $p \in \Gamma_{n}$. Furthermore as $\Omega$ is a bounded domain, the problem $(P)$ has at most one solution, see Remark $1.5(2)$. Thus $v$ is invariant under action of $R_{n}$. Namely we have $v\left(R_{n}(x)\right)=v(x)$ for every $x \in \Omega$. In particular if we choose $f \equiv 0$ to be the null function, then the graph of $v$ will be a complete minimal surface of $\mathbf{H}^{3}$. Note that we could also invoke Theorem 2.3 to insure existence and uniqueness of solution of the problem $(P)$.

LEMMA 3.8. Let $\Omega \subset \mathbf{R}^{2}$ be an unbounded domain. Suppose that $\partial \Omega$ is embedded and there exist a point $p \in \partial \Omega$ and a real number $\lambda>1$ such that $h_{\lambda, p}(\partial \Omega)=\partial \Omega$, where $h_{\lambda, p}$ is the homothety with respect to $p$, of ratio $\lambda$. Suppose also that $\bar{\Omega}$ is contained in a proper sector of $\mathbf{R}^{2}$ whose vertex is $\dot{p}$. Consider problem $(P)$ where $f \equiv 0$ is the null function. Let $\phi$ be any supersolution for $(P)$ such that $\phi\left(h_{\lambda, p}(x)\right)=\lambda \cdot \phi(x)$, $x \in \bar{\Omega}$, see Example 3.3 (2). Assume that the solution of Perron $v$ of $(P)$ relative to $\phi$ is continuous up to the boundary. Then, it follows that $v\left(h_{\lambda, p}(x)\right)=\lambda \cdot v(x)$ for every $x \in \Omega$. Consequently, the graph $S$ of $v$ is a complete minimal surface of $\mathbf{H}^{3}$ invariant by the hyperbolic isometry $h_{\lambda, p}: h_{\lambda, p}(S)=S$. 
Proof. Observe that $v(x)=0$ for each $x \in \partial \Omega$. We can assume that $p=0$ and then $h_{\lambda, p}(X)=h_{\lambda}(X)=\lambda \cdot X$ for every $X \in \overline{\mathbf{H}^{3}}$. Let us call $\mathcal{S}_{\phi}$ the family of subsolutions $u$ of $(P)$ such that $u \leq \phi$. By construction we have $v(x)=\sup \{u(x), u \in$ $\left.\mathcal{S}_{\phi}\right\}$ for any $x \in \bar{\Omega}$. Now observe that the minimal surface $\lambda . S$ is the vertical graph of the function $v_{\lambda}(x)=\lambda v\left(\frac{x}{\lambda}\right), x \in \bar{\Omega}$. By construction we have $v_{\lambda}(x)=0$ for any $x \in \partial \Omega$. We deduce that $v_{\lambda}$ is a subsolution for $(P)$ and $v_{\lambda} \leq \phi$ (since $v_{\lambda}(x)=$ $\left.\lambda v\left(\frac{x}{\lambda}\right) \leq \lambda \phi\left(\frac{x}{\lambda}\right)=\phi(x)\right)$. It follows that $v_{\lambda}(x) \leq v(x), x \in \bar{\Omega}$, from the very definition of $v$. On the other hand, the same argument applied with $1 / \lambda$ instead $\lambda$ shows that $v_{1 / \lambda}(x) \leq v(x), x \in \bar{\Omega}$. From which we deduce that $v_{\lambda}(x)=v(x), x \in \bar{\Omega}$.

Proposition 3.9. (Hyperbolic Surfaces) Let $\Omega \subset \mathbf{R}^{2}$ be an unbounded domain with $\partial \Omega$ embedded. Suppose that:

(i) $0 \in \partial \Omega$ and there exists $\lambda>1$ such that $\bar{\Omega}$ is invariant by the homothety $h_{\lambda}$ with respect to 0 whose ratio is $\lambda$.

(ii) For any $p \in \partial \Omega, p \neq 0$, there exists a hyperbolic catenoid $C$ such that $\partial_{\infty} C \cap$ $\Omega=\emptyset, p \in \partial_{\infty} C$ and such that the segment joining the centers of the two components of $\partial_{\infty} C$ intersects $\Omega$.

(iii) $\bar{\Omega}$ is contained in a proper sector of $\mathbf{R}^{2}$ whose vertex is 0 .

Consider problem $(P)$ where $f$ is the vanishing function on $\partial \Omega$. Then $(P)$ has a solution $v$ which is invariant under the homothety $h_{\lambda}$. Consequently, the graph $S$ of $v$ is a complete minimal surface of $\mathbf{H}^{3}$ invariant under the discrete group of hyperbolic isometries $\left\{h_{\lambda}^{q}, q \in \mathbf{Z}\right\}$.

Proof. Observe that as $\bar{\Omega}$ is contained in a proper sector, the problem $(P)$ admits a supersolution $\phi$, such that $\phi(\lambda x)=\lambda \cdot \phi(x), x \in \bar{\Omega}$, see Example 3.3 (2). Furthermore the condition ( $i i)$ implies that each point $p \in \partial \Omega, p \neq 0$, has a superior barrier (for $(P))$. As $\partial \Omega$ is convex at the origin 0 , we know that 0 has a barrier for $(P)$, see Remark 1.9 (1). Note that the null function is an inferior barrier for any point $p \in \partial \Omega$. We conclude that any point $p \in \partial \Omega$ has a barrier for $(P)$. Therefore, if $v$ is the solution of Perron (relative to $\phi$ ) of $(P)$ it follows that $v$ is continuous up to the boundary and $v_{\mid \partial \Omega}=0$, see Theorem 3.4. Furthermore, Lemma 3.8 shows that $v(\lambda \cdot x)=\lambda \cdot v(x)$ for any $x \in \bar{\Omega}$.

REMARK 3.10. In the context of Proposition 3.9, suppose that $\Omega$ is $C^{2}$. Let $R>0$ and set $\Gamma=\{p \in \partial \Omega, R \leq|p| \leq \lambda . R\}$. Let $k(x)$ be the euclidean curvature of $\Gamma$ with respect to the interior normal orientation. Let $\theta \in] 0, \pi[$ be the angle of any proper sector containing $\bar{\Omega}$ whose vertex is 0 . Suppose that $\theta$ is small enough so that the condition ( $i i)$ is satisfied for $S_{\theta}$. Consequently, the condition (ii) is achieved (for $\Omega$ ) if it is assumed that $|k(x)|$ is small enough.

In the same way, we can prove analogous statements for parabolic minimal surfaces, the arguments are essentially the same. For this reason we just state the results without give a proof.

LeMmA 3.11. Let $\Omega \subset \mathbf{R}^{2}$ be an unbounded domain. Suppose that $\partial \Omega$ is embedded and there exists a non null vector $\xi \in \mathbf{R}^{2}$ such that $T(\bar{\Omega})=\bar{\Omega}$, where $T$ is the euclidean translation $T(x)=x+\xi, x \in \mathbf{R}^{2}$. Suppose also that $\bar{\Omega}$ is contained in a band of $\mathbf{R}^{2}$ invariant under T. Consider problem $(P)$ where $f \equiv 0$ is the null function. Let $\phi$ be any supersolution for $(P)$ such that $\phi(T(x))=\phi(x), x \in \bar{\Omega}$ (see Example 3.3 (2) or (3)). Assume that the solution of Perron $v$ of $(P)$ relative to $\phi$ is continuous up 
to the boundary. Then we have $v(T(x))=v(x)$ for every $x \in \Omega$. Consequently, the graph $S$ of $v$ is a complete minimal surface of $\mathbf{H}^{3}$ invariant by the parabolic isometry $T: T(S)=S$.

Proposition 3.12. (Parabolic Surfaces) Let $\Omega \subset \mathbf{R}^{2}$ be an unbounded domain with $\partial \Omega$ embedded. Suppose that:

(i) There exists a non null vector $\xi \in \mathbf{R}^{2}$ such that $\bar{\Omega}$ is invariant by the horizontal translation $T(x)=x+\xi$

(ii) $\bar{\Omega}$ is contained in a band invariant under $T$.

(iii) For any $p \in \partial \Omega, p \neq 0$, there exists a hyperbolic catenoid $C$ such that $\partial_{\infty} C \cap$ $\Omega=\emptyset, p \in \partial_{\infty} C$ and such that the segment joining the centers of the two components of $\partial_{\infty} C$ intersects $\Omega$.

Consider problem $(P)$ where $f$ is the vanishing function on $\partial \Omega$. Then $(P)$ has a solution $v$ which is invariant under the horizontal translation $T$. Consequently, the graph $S$ of $v$ is a complete minimal surface of $\mathbf{H}^{3}$ invariant under the discrete group of parabolic isometries $\left\{T^{q}, q \in \mathbf{Z}\right\}$.

4. Graphs over unbounded domains. For the whole section we design by $B$ the open band $B=\left\{(x, y) \in \mathbf{R}^{2}, 0<y<1\right\}$. Observe that for any open band $C$ in $\mathbf{R}^{2}=\partial_{\infty} \mathbf{H}^{3}$ there exists an isometry of $\mathbf{H}^{3}$ sending $C$ on $B$. Therefore there is no loss of generality in just consider the band $B$.

TheOREM 4.1. Let $\Omega \subset B$ be a convex domain. Let $f: \partial \Omega \rightarrow\left[0,+\infty\left[\right.\right.$ be a $C^{0}$ function. Then problem $(P)$ has a solution. That is $f$ admits a minimal extension on $\Omega$.

Proof. For any $n \in \mathrm{N}$ set $\Omega_{n}=\{(x, y) \in \Omega,-n<x<n\}$. Note that $\Omega_{n}$ is a bounded and convex domain. Let $f_{n}: \partial \Omega_{n} \rightarrow[0,+\infty[$ be any continuous function such that $\left(f_{n}\right)_{\mid \partial \Omega}=f$. Let us call $u_{n}$ the unique minimal extension of $f_{n}$ on $\Omega_{n}$, see Theorem 2.3. Consider now a hyperbolic catenoid $C_{n}$ such that $\partial_{\infty} C_{n}$ has a component on each side of the band $B$. Up to a homothety, we can assume that the vertical projection of $C_{n}$ on $\mathbf{R}^{2}=\partial_{\infty} \mathbf{H}^{3}$, contains $\bar{\Omega}_{n}$ in its interior. Observe that there exists a positive number $t_{n}$ such that the vertical translated $\tilde{C}_{n}=C_{n}+\left(0,0, t_{n}\right)$ stays strictly above the graph of $f$. We deduce with the Maximum Principle that if $k>>n$ is sufficiently big, the graph of $u_{k}$ over $\Omega_{n}$ stays below $\tilde{C_{n}}$. This gives uniform upper estimate for the sequence $\left(u_{k}\right)$ over $\Omega_{n}$ (at least for $k$ big enough). Now, for any $\varepsilon>0$ set $\Omega_{n, \varepsilon}=\left\{p \in \Omega_{n}, d\left(p, \partial \Omega_{n}\right)>\varepsilon\right\}$. Let $p \in \Omega_{n, \varepsilon}$ be any point and let $\Pi_{p}$ be any hemisphere in $\mathbf{H}^{3}$ centered at $p$ such that $\partial_{\infty} \Pi_{p} \subset \Omega_{n, \varepsilon}$. As $\Pi_{p}$ is a minimal surface in $\mathbf{H}^{3}$, the Maximum Principle shows that the graph of any function $u_{k}$ stays above $\Pi_{p}$ for $k>>n$ big enough. This gives uniform lower $C^{0}$ estimate for the sequence $\left(u_{k}\right)$ on $\Omega_{n, \varepsilon}$. As the family $\overline{\Omega_{n, \varepsilon}}$ is a compact exhaustion of $\Omega$, we infer, according to Theorem 1.3, that there is a subsequence of $\left(u_{k}\right)$ which converges on $\Omega$ for the $C^{2}$ topology to a $C^{2}$ function $u$ satisfying (Eq 1-1). As $\Omega$ is convex we know that each point of $\partial \Omega$ has a barrier, see Remark 1.9 (1). From which we deduce that $u$ is $C^{0}$ up to $\partial \Omega$ and $u=f$ on $\partial \Omega$ as in the proof of Theorem 3.4.

Corollary 4.2. Let $\Omega \subset B$ be a convex domain. Let $f: \partial \Omega \rightarrow[0,+\infty[$ be a $C^{0}$ function. Assume that $f$ is bounded and uniformly continuous. Then problem $(P)$ admits an unique solution $u$. Furthermore $u$ is also bounded and uniformly continuous on $\Omega$. 
Proof. We know by Theorem 4.1 that problem $(P)$ admits a solution. The uniqueness arises from Corollary 1.11. Furthermore, Theorem 1.7 shows that $u$ is bounded. Finally, we deduce from Corollary 1.8 that $u$ is uniformly continuous.

Theorem 4.3. (Maximum Principle in a band) Let $\Omega \subset B$ be a domain and let $f: \partial \Omega \rightarrow\left[0,+\infty\left[\right.\right.$ be a continuous function such that $f(p) \leq u_{B}(p)$ for every $p \in \partial \Omega$ (see Example 3.3 (2) for the definition of $u_{B}$ ). Then any minimal extension $F$ over $\Omega, F_{\mid \partial \Omega}=f$, satisfies $F \leq u_{B}$.

Proof. Let us consider the following problem $(\hat{P})$ :

$$
(\hat{P}) \quad\left\{\begin{aligned}
\sum_{i, j=1}^{n}\left(\delta_{i j}-\frac{u_{i} u_{j}}{1+|D u|^{2}}\right) u_{i j}+\frac{n}{u} & =0 \text { on } B \\
u_{\mid \partial B} & =0 \\
u & \in C^{2}(B) \cap C^{0}(\bar{B})
\end{aligned}\right.
$$

Define $v(p)=\sup \left(F(p), u_{B}(p)\right), p \in \Omega$ and $v(p)=u_{B}(p)$ for any $p \in \bar{B}-\Omega$. As the graph of $f$ stays below the graph of $u_{B}$ we deduce that $v$ is a continuous function on $\bar{B}$ with $v_{\mid \partial B} \equiv 0$. It is easy to see that $v$ is a subsolution for problem $(\hat{P})$. Furthermore, using a hyperbolic catenoid as in Example 3.3 (3) we can construct a supersolution $\phi$ for $(\hat{P})$ whose graph stays above the graphs of $u_{B}$ and $F$. We deduce that $v \leq \phi$. Set $\mathcal{S}_{\phi}=\left\{u \in C^{0}(\bar{B})\right.$, subsolution of $\left.(\hat{P}), u \leq \phi\right\}$. As in Theorem 3.4 we get that $U(p)=\sup _{u \in \mathcal{S}_{\phi}}\{u(p)\}$ is a solution of $(\hat{P})$. As problem $(\hat{P})$ has an unique solution, see Corollary 4.2 , we deduce that $U \equiv u_{B}$. From which we conclude that $F \leq u_{B}$ for any minimal extension $F$ of $f$ on $\Omega$.

DEFINITION 4.4. For every $\theta \in] 0, \pi\left[\right.$ we will design by $S_{\theta}$ any open sector of $\mathbf{R}^{2}=\partial_{\infty} \mathbf{H}^{3}$ whose angle at the vertex is $\theta$.

Theorem 4.5. (Maximum Principle in a proper sector) Let $u_{\theta} \in C^{0}\left(\bar{S}_{\theta}\right) \cap$ $C^{2}\left(S_{\theta}\right)$ be a function satisfying $\mathcal{D}\left(u_{\theta}\right)=0$ and $u_{\theta} \equiv 0$ on $\partial S_{\theta}$. Let $\Omega \subset S_{\theta}$ be a domain and $f: \partial \Omega \rightarrow\left[0,+\infty\left[\right.\right.$ be a continuous function such that $f(p) \leq u_{\theta}(p)$ for every $p \in \partial \Omega$. Then any minimal extension $F$ of $f$ on $\Omega$ satisfies $F \leq u_{\theta}$.

Proof. We can assume that $S_{\theta}=\left\{p \in \mathbf{R}^{2}, 0<\arg (p)<\theta\right\}$ (up to an isometry of $\mathbf{H}^{3}$ ). Let $p_{0} \in \mathbf{R}^{2}$ be any point such that $\arg \left(p_{0}\right)=\frac{\theta}{2}+\pi$. That is $p_{0} \notin \bar{S}_{\theta}$ and $p_{0}$ lies on the median line of $S_{\theta}$. Let $\Pi_{0} \subset \mathbf{H}^{3}$ be a hemisphere centered at $p_{0}$ such that $\partial_{\infty} \Pi_{0} \cap \bar{S}_{\theta}=\emptyset$. Observe that $\Pi_{0}$ is a geodesic plane of $\mathbf{H}^{3}$ and that reflection with respect to $\Pi_{0}$ is an isometry of $\mathbf{H}^{3}$. If $N$ is any part of $\mathbf{H}^{3}$ or $\mathbf{R}^{2}$ we design by $N^{*}$ the reflected of $N$ with respect to $\Pi_{0}$.

Note that $\bar{S}_{\theta}{ }^{*}$ is a compact and $C^{0}$-convex domain in $\mathbf{R}^{2}$. Let $M \subset \mathbf{H}^{3}$ be the graph of $u_{\theta}$ over $S_{\theta}$. Therefore $M^{*}$ is a minimal surface in $\mathbf{H}^{3}$ whose asymptotic boundary $\left(\partial{\overline{S_{\theta}}}^{*}\right)$ is compact and convex. We infer from Theorem 2.4 that $M^{*}$ is a vertical graph. Now let $N \subset \mathbf{H}^{3}$ be the graph of $F$, where $F$ is any minimal extension of $f$ on $\Omega$. It follows from hypothesis that $N^{*}$ is a minimal surface whose boundary stays in the closure of the "bounded" component of $\mathbf{H}^{3}-M^{*}$. Suppose, by absurd, that $N^{*}$ does not stay in this bounded component. Let $p \in S_{\theta}^{*}$ be any point. Consider the homotheties $h_{p, \lambda}$ from $p$, where $\lambda>0$. As $M^{*}$ and $N^{*}$ are compact in $\overline{\mathbf{H}^{3}}$ there is a real number $\lambda>1$ such that $h_{p, \lambda}\left(M^{*}\right)$ and $N^{*}$ are tangent at some point and $h_{p, \lambda}\left(M^{*}\right)$ stays above $N^{*}$. But this gives a contradiction with Maximum Principle since both 
surfaces are minimal in $\mathbf{H}^{3}$. We deduce that $N^{*}$ stays below $M^{*}$. Reflecting with respect to $\Pi_{0}$ we conclude that $F \leq u_{\theta}$ for any minimal extension $F$ of $f$ on $\Omega$.

Proposition 4.6. For every $\theta \in] 0, \pi[$, there is at most one connected complete minimal surface $M$ in $\mathbf{H}^{3}$ which is properly immersed such that its asymptotic boundary is $\partial S_{\theta} \cup\{\infty\}$.

In particular if $\Omega=S_{\theta}$ and $f \equiv 0$ is the null function on $\partial \Omega$, the problem $(P)$ has at most one solution.

Proof. We use the same notations and constructions as in the proof of Theorem 4.5. Let $M$ and $N$ two minimal surfaces in $\mathbf{H}^{3}$ satisfying the hypothesis of Proposition 4.6. We get that $M^{*}$ and $N^{*}$ are graphs over the convex and bounded domain $S_{\theta}^{*}$. Then using the homotheties $h_{p, \lambda}$ as in the proof of Theorem 4.5, we can show that $M^{*}$ stays below and above $N^{*}$. We deduce that $M^{*}=N^{*}$. Reflecting with respect to $\Pi_{0}$ we get $M=N$.

REMARK 4.7. 1) We will see in the Appendix that it does exist for any $\theta \in] 0, \pi[$ a solution $u_{\theta}$ for problem $(P)$, where $\Omega=S_{\theta}$ and $f \equiv 0$ is the null function on $\partial \Omega$. It follows from Proposition 4.6 that $(P)$ has an unique solution. Furthermore we will see that the graph of $u_{\theta}$ has an important geometric property.

2) Let $\Omega \subset S_{\theta}$ be a $C^{0}$-convex domain in a proper sector, $\left.\theta \in\right] 0, \pi[$. Let $f$ : $\partial \Omega \rightarrow\left[0,+\infty\left[\right.\right.$ be a continuous function such that $f \leq u_{\theta}$ on $\partial \Omega$. Observe that $u_{\theta}$ is a supersolution for problem $(P)$. We can deduce, using Perron process as in Theorem 3.4 , that problem $(P)$ admits a solution. That is $f$ has a minimal extension on $\Omega$. Also, let $\Omega=S_{\theta}$ be a proper sector and let $f: \partial S_{\theta} \rightarrow[0,+\infty$ [ be a continuous bounded function. Then, there exists a positive constant $c$ such that $f \leq u_{\theta}+c$ on $\partial S_{\theta}$. Therefore the function $u_{\theta}+c$ is a supersolution for the problem $(P)$ and we deduce in the same way that $(P)$ admits a solution.

3) Consider the limit case where $\theta=\pi$, that is $S_{\pi}$ is a half-plane. let $f: \partial \Omega \rightarrow$ $[0,+\infty[$ be any continuous function. Then problem $(P)$ admits no solution. Indeed, let $p \in S_{\pi}$ be any point. Let $\Pi \subset \mathbf{H}^{3}$ be any geodesic plane such that $\partial_{\infty} \Pi \subset S_{\pi}$ is a circle in $S_{\pi}$ and $p$ belongs to the open disk bounded by $\partial_{\infty} \Pi$. We get from the Maximum Principle that $\Pi$ stays below any minimal extension $F$ of $f$. Therefore, as the radius of $\Pi$ can be made as large as we want, we deduce that $F(p)=+\infty$ for any $p \in S_{\pi}$ and any solution $F$ of $(P)$, which is absurd. In fact, this argument holds for any dimension.

Appendix. For sake of completeness, we give a proof for existence of some minimal surfaces in $\mathbf{H}^{3}$. There were studied in the paper of M. Gomes, J. Ripoll and L. Rodriguez entitled: On surfaces of constant mean curvature in hyperbolic space, preprint 1985, IMPA.

From now on, we design by $\left.S_{\theta}, \theta \in\right] 0, \pi\left[\right.$, the open sector of $\mathbf{R}^{2}$ whose vertex is $q=(-1,0,0)$, with angle $\theta$ and whose median line is the real ray $[-1,+\infty[$.

That is:

$$
S_{\theta}=\left\{p \in \mathbf{R}^{2},-\frac{\theta}{2}<\arg (1+p)<\frac{\theta}{2}\right\}
$$

Proposition A.1. Let $\theta \in] 0, \pi[$ be any number. Then there exists an unique complete minimal surface $M_{\theta}$ properly immersed in $\mathbf{H}^{3}$, whose asymptotic boundary 
is the boundary of $S_{\theta}$. Moreover this surface is a graph over $S_{\theta}$ and is invariant under the homotheties $h_{q, \lambda}$, with fixed point $q=(-1,0,0)$, for any $\lambda>0$.

Proof. We will construct a solution for problem $(P)$ where $\Omega=S_{\theta}$ and $f \equiv 0$ on $\partial \Omega$. For this we first provide a supersolution $\phi$. To fix notations, let us say that the real ray $\left[-1,+\infty\right.$ [ is supported by the $x_{1}$-axis. Let $\Pi$ be the vertical half-plane through $(0,0,0)$ orthogonal to the $x_{1}$-axis, that is $\Pi=\left\{X \in \mathbf{H}^{3}, x_{1}=0\right\}$. Consider the following $C^{2}$-curve on $\left.\Pi: \gamma(t)=(0, r \cos (t), a+r \sin (t)), t \in\right] 0, \pi$ [ where $a>0$ is a real number to be defined later, and $r>0$ is a fixed number. Note that the image of $\gamma$ is a vertical semi-circle $\Gamma$ of radius $r$. Then we choose $r$ big enough so that the points $\pm(0, r, 0) \notin \overline{S_{\theta}}$ and the segment joining those two points intersects $S_{\theta}$. Let $\mathcal{C} \subset \mathbf{H}^{3}$ be the "cone" over $\Gamma$ with respect to the point $q=(-1,0,0)$. That is $\mathcal{C}=\cup_{\lambda>0} h_{q, \lambda}(\Gamma)$ where $h_{q, \lambda}(X)=\lambda \cdot(X+(1,0,0))+(-1,0,0)$ for any $\lambda>0$ and every $X \in \overline{\mathbf{H}^{3}}$. Observe that $\mathcal{C}$ is a $C^{2}$ surface, and is the graph of a $C^{2}$ function $\phi$ defined in a sector containing $S_{\theta}$ in its interior. In order to compute the hyperbolic mean curvature $H_{H}$ of $\mathcal{C}$ we first evaluate its euclidean mean curvature $H_{E}$ both in relation to the normal orientation corresponding to increasing $z$. For this we use the following parametrization of $\mathcal{C}=X(] 0, \pi[\times] 0,+\infty[)$ where:

$$
X(t, \lambda)=\lambda \cdot[(0, r \cos (t), a+r \sin (t))+(1,0,0)]+(-1,0,0), t \in] 0, \pi[, \lambda>0 .
$$

A straightforward calculation shows that the euclidean mean curvature $H_{E}$ of $\mathcal{C}$ is:

$$
H_{E}(t, \lambda)=-\frac{1+r^{2}+2 a r \sin (t)+a^{2}}{2 \lambda r\left[1+(r+a \sin (t))^{2}\right]^{3 / 2}} .
$$

We have the following identities:

$$
\begin{aligned}
\mathcal{D}(\phi) & =\sqrt{1+|D \phi|^{2}}\left[\operatorname{div}\left(\frac{D \phi}{\sqrt{1+|D \phi|^{2}}}\right)+\frac{2}{\phi \sqrt{1+|D \phi|^{2}}}\right] \\
& =\left[2 H_{E}+\frac{2 N_{3}}{x_{3}}\right] \cdot \frac{1}{N_{3}} \\
& =2 \frac{\sqrt{1+|D \phi|^{2}}}{\phi} H_{H}
\end{aligned}
$$

where $D \phi$ is the euclidean gradient vector of $\phi, N_{3}=\frac{1}{\sqrt{1+|D \phi|^{2}}}$ is the third component of the upper unit euclidean normal vector field $N$ along $\mathcal{C}$. From which we infer:

$$
\begin{aligned}
\mathcal{D}(\phi)<0 & \Leftrightarrow \frac{2 \sin (t)}{\lambda(a+r \sin (t)) \sqrt{1+(r+a \sin (t))^{2}}}<\frac{1+r^{2}+2 a r \sin (t)+a^{2}}{\lambda r\left[1+(r+a \sin (t))^{2}\right]^{3 / 2}} \\
& \Leftrightarrow 2 \sin (t) r\left[1+(r+a \sin (t))^{2}\right]<(a+r \sin (t))\left(1+r^{2}+2 a r \sin (t)+a^{2}\right)
\end{aligned}
$$

Clearly, the last inequality will be satisfied if we choose $a>0$ big enough. Therefore, $\phi$ is a supersolution for problem $(P)$. It follows from Theorem 3.4 that $(P)$ has a solution $u_{\theta}$. It is inferred from Proposition 4.6 that $u_{\theta}$ is the unique solution of $(P)$. Let $M_{\theta} \subset \mathbf{H}^{3}$ be the graph of $u_{\theta}$. Observe that for any $\lambda>0$ the surface $h_{q, \lambda}\left(M_{\theta}\right)$ is also a minimal graph over $S_{\theta}$ with zero boundary value data. Uniqueness of such a graph yields $h_{q, \lambda}\left(M_{\theta}\right)=M_{\theta}$ for any $\lambda>0$. More precisely, $M_{\theta}$ is invariant under the homotheties with respect to $q=(-1,0,0)$. 
Proposition A.2. Consider the horizontal open band $B=\left\{\left(x_{1}, x_{2}\right) \in \mathbf{R}^{2},-1<\right.$ $\left.x_{2}<1\right\}$. There is an unique connected complete minimal surface $M$ properly immersed in $\overline{\mathbf{H}^{3}}$ such that:

(i) $\partial_{\infty} M=\partial B \cup\{\infty\}$

(ii) $M$ is globally invariant under any horizontal translation leaving $B$ invariant. More precisely, $T_{x_{1}}(M)=M$ for every $x_{1} \in \mathbf{R}$, where $T_{x_{1}}$ is the horizontal translation defined by $T_{x_{1}}(X)=X+\left(x_{1}, 0,0\right)$.

Furthermore, $M$ is a vertical graph over the band $B$.

Proof. Consider the vertical half-plane $\Pi=\left\{x_{1}=0\right\}$ in $\mathbf{H}^{3}$. Let $M \subset \mathbf{H}^{3}$ be a complete minimal surface as in Proposition A.2. Observe that $\Pi$ and $M$ are transverse and $\Pi \cap M$ is a connected $C^{2}$ curve $\Gamma$. Now we use the following notations: $y=x_{2}$ and $z=x_{3}$. Let $\left.\gamma(t)=(0, y(t), z(t)), t \in\right] 0,1[$ be a regular parametrization of $\Gamma$. We can assume $\lim _{t \rightarrow 0} y(t)=-1$ and $\lim _{t \rightarrow 1} y(t)=1$. By hypothesis the surface $M$ is obtained translating horizontally $\Gamma$. That is $M=\cup_{x \in \mathbf{R}} T_{x}(\Gamma)$ where $T_{x}(X)=X+(x, 0,0)$ for every $X \in \overline{\mathbf{H}^{3}}$. Consider the following parametrization of $M=X(] 0,1[\times \mathbf{R})$ where:

$$
X(t, x)=(x, y(t), z(t)), t \in] 0,1[, x \in \mathbf{R} .
$$

Let $N(t, x)=N(t)=\frac{1}{\sqrt{y^{\prime 2}+z^{\prime 2}}} \cdot\left(0,-z^{\prime}(t), y^{\prime}(t)\right)$ be an unit euclidean normal vector field along $M$. The euclidean mean curvature of $M$ with respect to the normal field $N$ is:

$$
H_{E}(t, x)=H_{E}(t)=\frac{\left(z^{\prime \prime} y^{\prime}-z^{\prime} y^{\prime \prime}\right)}{2\left(y^{\prime 2}+z^{\prime 2}\right)^{3 / 2}}(t) .
$$

Let $H_{H}$ be the hyperbolic mean curvature with respect to the normal field $N$. We have:

$$
2 H_{E}=\frac{2}{z}\left(H_{H}-N_{3}\right)
$$

where $N_{3}$ is the third component of $N$. Thus

$$
H_{H} \equiv 0 \Leftrightarrow z^{\prime \prime} y^{\prime}-z^{\prime} y^{\prime \prime}=-2 \frac{y^{\prime}\left(y^{\prime 2}+z^{\prime 2}\right)}{z} .
$$

Observe that the previous differential equation is invariant by homotheties in $y$ and $z$ coordinates. If we assume that $\Gamma$ is a graph with respect to $y$, we have $\gamma(y)=$ $(0, y, z(y))$. Therefore

$$
H_{H} \equiv 0 \Leftrightarrow z^{\prime \prime}=-2 \frac{\left(1+z^{\prime 2}\right)}{z} .
$$

Let us show that equation $(*)$ admits a "complete" solution of the following form: $z=z(y), y \in]-1,1\left[\right.$, with $\lim _{y \rightarrow \pm 1} z(y)=0$. For this we will use the same technics as in [SE-To, 2] or [SE-To, 3]. First using Picard theorem we get, for every $\tau>0$, an unique solution $z_{\tau}$ of $(*)$ defined on an interval ] $-y_{0}, y_{0}$ [ such that $z_{\tau}(0)=\tau$ and $z_{\tau}^{\prime}(0)=0$. Observe that the function $Z(t)=z(-t)$ satisfies the same equation $(*)$ with same initial conditions. Hence $z_{\tau}(-t)=z_{\tau}(t)$, that is the graph of $z_{\tau}$ is symmetric with respect to the $z$-axis. Now as $z^{\prime \prime}<0$ on ] $-y_{0}, y_{0}$ [it follows that $z^{\prime}<0$ on $] 0, y_{0}[$. Thus $z_{\tau}$ and $z_{\tau}^{\prime}$ has a limit at $y_{0}$. Clearly $\left.z_{\tau}\left(y_{0}\right) \in\right] 0, \tau\left[\right.$. Suppose that $z_{\tau}^{\prime}\left(y_{0}\right)=-\infty$. Let $P \subset \mathbf{H}^{3}$ be the vertical half-plane $\left\{y=y_{0}\right\}$. Let $\Gamma_{\tau}$ be the graph of $z_{\tau}$. Let us call $M_{\tau}=\cup_{x \in \mathbf{R}}\left(T_{x}\left(\Gamma_{\tau}\right)\right)$ the (minimal) translation surface generated by $\Gamma_{\tau}$. Note that $P$ and $M_{\tau}$ are tangent at $\left(0, y_{0}, z\left(y_{0}\right)\right)$ and $M_{\tau}$ stays in one side of $P$. This 
gives a contadiction with the Maximum Principle with boundary. We deduce that $-\infty<z_{\tau}^{\prime}\left(y_{0}\right)<0$. Using again the Picard theorem this allows us to extend the solution $z_{\tau}$ to a larger interval. As we have $z_{\tau}^{\prime \prime}<0$ we get a solution satisfying: $z_{\tau}(0)=\tau, z_{\tau}^{\prime}(0)=0, z_{\tau}$ is defined in some interval $]-y_{\tau}, y_{\tau}\left[\right.$ with $\lim _{y \rightarrow \pm y_{\tau}} z(y)=0$. Observe that the translation surface $M_{\tau}$ is a complete minimal surface in $\mathbf{H}^{3}$, which is a graph over a band $B_{\tau}$. Moreover we have $\partial_{\infty} M_{\tau}=\partial B_{\tau} \cup\{\infty\}$. At last, using the homothetic curves $\alpha \cdot\left(y, z_{\tau}(y)\right)$, varying $\alpha$, we can find an $\alpha>0$ such that $B_{\tau}$ is precisely the band $B$. This proves the existence part in the statement of Proposition A.2. Let us call more briefly $M$ this surface and $\Gamma$ the corresponding curve.

Now we prove uniqueness. Let $M_{1} \subset \mathbf{H}^{3}$ be another surface satisfying the assumptions of Proposition A.2. Observe that $M_{1} \cap \Pi$ is a connected $C^{2}$-curve $\Gamma_{1}$ whose boundary points are $\pm(0,1,0)$ as well as $\Gamma$. Suppose the two curves $\Gamma$ and $\Gamma_{1}$ are not identical. Then there is a real number $\alpha>0$ such that the homothetic curve $\alpha \cdot \Gamma$ and $\Gamma_{1}$ are tangent at some point. Thus those curves satisfy the same differential equation (*) with same initial conditions. This gives a contradiction and shows uniqueness in the statement.

\section{REFERENCES}

[An, 1] M. Anderson, Complete minimal varieties in hyperbolic space, Invent. Math., 69 (1982), pp. $477-494$.

[An, 2] M. ANDERson, Complete minimal hypersurfaces in hyperbolic n-manifolds, Comment. Math. Helv., 58 (1983), pp. 264-290.

[Be] L. BERs, Isolated singularities of minimal surfaces, Ann. of Math., 53 (1952), pp. 364-386.

[Co-Hi] R. Courant And D. Hilbert, Methods of Mathematical Physics 2, Inter-Science, NewYork, 1962.

[Gi-Tr] D. Gilbarg and N. S. Trudinger, Elliptic Partial Differential Equations of Second Order, second edition, Springer-Verlag, 1983.

[Je-Se, 1] H. JEnkins AND J. SERRIN, The Dirichlet problem for the minimal surface equation in higher dimensions, J. Reine Angew. Math., 229 (1968), pp. 170-187.

[Je-Se, 2] H. JENKINS AND J. SERRIN, Variational problems of minimal surface type. Boundary value problems for the minimal surface equation II, Arch. Rational Mech. Analysis, 21 (1963), pp. 321-342.

[La-Ro] R. LANGEVIN AND H. ROSENBERG, A maximum principle at infinity for minimal surfaces and applications, Duke Math. J., 57 (1988), pp. 819-828.

[Li,1] F. H. LIN, On the Dirichlet problem for minimal graphs in hyperbolic space, Invent. Math., 96 (1989), pp. 593-612.

[Li,2] F. H. LIN, Asymtotic behavior of area-minimizing currents in hyperbolic space, Comm. Pure and Appl. Math., 42 (1989), pp. 229-242.

[Me-Ro] W. MEeKs III AND H. ROSENBERG, The maximum principle at infinity for minimal surfaces in flat 3-manifolds, Comm. Math. Helv., 65 (1990), pp. 255-270.

[Pr-We] M. H. Protter and H. F. Weinberger, Maximum Principles in Differential Equations, Elglewood Cliffs, ed., New Jersey Prentice Hall, 1967.

[Ro-SE] H. Rosenberg and R. SA EARP, The Dirichlet problem for minimal surface equation on unbounded planar domains, J. Math. Pures Appl., 68 (1989), pp. 163-183.

[SE-To, 1] R. SA EARP AND E. TOUBIANA, Some applications of maximum principle to hypersurfaces theory in euclidean and hyperbolic space, in New Approaches in Nonlinear Analysis, T. M. Rassias, ed., Hadronic Press Inc, Florida, USA, 1999, pp. 183-202.

[SE-To, 2] R. SA EARP AND E. TOUBIANA, Sur les surfaces de Weingarten spéciales de type minimal, Boletim Soc. Bras. Mat., 26:2 (1995), pp. 129-148.

[SE-To, 3] R. Sa EARP AND E. Toubiana, Classification des surfaces de type Delaunay, American J. of Math., 121 (1999), pp. 671-700.

[Si] L. Simon, Equations of mean curvature type in 2 independent variables, Pacific J. of Math., 69:1 (1977), pp. 245-268. 
[So] M. SoRET, Deformations de surfaces minimales, These de Doctorat, Univ. de Paris VII, 1993. 
